\title{
Distributed Transient Frequency Control for Power Networks with Stability and Performance Guarantees
}

\author{
Yifu Zhang ${ }^{\text {a }}$ Jorge Cortés ${ }^{\text {a }}$ \\ ${ }^{a}$ Department of Mechanical and Aerospace Engineering, University of California, San Diego, \{yifuzhang,cortes\}@ucsd.edu
}

\begin{abstract}
This paper proposes a distributed strategy regulated on a subset of individual buses in a power network described by the swing equations to achieve transient frequency control while preserving asymptotic stability. Transient frequency control refers to the ability to maintain the transient frequency of each bus of interest in a given safe region, provided it is initially in it, and ii) if it is initially not, then drive the frequency to converge to this region within a finite time, with a guaranteed convergence rate. Building on Lyapunov stability and set invariance theory, we formulate the stability and the transient frequency requirements as two separate constraints for the control input. Our design synthesizes a controller that satisfies both constraints simultaneously. The controller is distributed and Lipschitz, guaranteeing the existence and uniqueness of the trajectories of the closed-loop system. We further bound its magnitude and demonstrate its robustness against measurement inaccuracies. Simulations on the IEEE 39-bus power network illustrate our results.
\end{abstract}

Key words: Power networks, power systems stability, transient frequency, distributed control.

\section{Introduction}

In power system planning and operation against contingencies (e.g., generator loss, transmission line tripping, unexpected power demands), to avoid the system from running underfrequency or to help the network recover from it, load shedding and curtailment are commonly employed to balance supply and demand. However, due to inertia, it takes some time for the energy resources to re-enter a safe frequency region until the power network eventually converges to steady state. Hence, during transients, generators are still in danger of reaching their frequency limits and being tripped, which may in turn cause blackouts. This phenomenon tends to happen more frequently in modern power networks due to low inertia and highly-dynamic units. Therefore, there is a need to analyze the transient behavior of power networks and design controllers that ensure the safe evolution of the system.

Literature review. Transient stability refers to the ability of power networks to maintain synchronism after being subjected to a disturbance, see e.g., [Kundur et al., 2004]. Many works, see e.g., [Chiang, 2011, Dörfler et al., 2013, Menck et al., 2014], provide conditions to ensure synchronicity and investigate their relationship with the topology of the power network. However, even if network synchronism holds, system transient trajectory may enter unsafe regions, e.g., transient frequency may violate individual generator's frequency limits, causing generator failure and leading to blackouts [Kundur, 1994]. Hence, various techniques have been proposed to improve transient behavior. These include resource re-dispatch with transient stability constraints Alam and Makram, 2006, Nguyen et al. 2011]; thyristor-controlled series

\footnotetext{
* A preliminary version has been accepted at the IEEE Conference on Decision and Control as [Zhang and Cortés, 2018]. This work was supported by NSF award CNS-1329619 and AFOSR Award FA955015-1-0108.
}

capacitor compensation to optimize transmission impedance and keep power transfer constant [Grunbaum and Pernot, 2001]; the use of power system stabilizers to damp out low frequency intermachine oscillations [Mahmud et al., 2014], and placing virtual inertia in power networks to mitigate transient effects [Borsche et al. 2015, Poolla et al., 2017]. While these approaches have a qualitative effect on transient behavior, they do not offer strict guarantees as to whether the transient frequency stays within a specific region. Furthermore, the approach by Borsche et al. [2015] requires a priori knowledge of the time evolution of the disturbance trajectories and an estimation of the transient overshoot. Alternative approaches rely on the idea of identifying the disturbances that may cause undesirable transient behaviors using forward and backward reachability analysis, see e.g., [Althoff. 2014, Chen and Domínguez-García, 2012, Choi et al., 2016] and our previous work [Zhang and Cortés, 2017]. The lack of works that provide tools for transient frequency control motivates us here to design feedback controllers for the generators that guarantee simultaneously the stability of the power network and the desired transient frequency behavior. Our design is inspired by the controller-design approach to safety-constrained systems taken by Ames et al. [2017], where the safety region is encoded as the zero-sublevel set of a barrier function and safety is ensured by constraining the evolution of the function along the system trajectories.

Statement of contributions. The main result of the paper is the synthesis of a Lipschitz continuous, distributed controller, available at specific individual generator nodes, that satisfies the following requirements (i) renders the closed-loop power network asymptotically stable; (ii) for each controlled generator node, if its initial frequency belongs to a desired safe frequency region, then its frequency trajectory stays in it for all subsequent time; and (iii) if, instead, its initial frequency does not belong to the safe region, then the frequency trajectory enters it in finite time, 
and once there, never leaves. Our technical approach to achieve this combines Lyapunov stability and set invariance theory. We first show that requirement (iii) automatically holds if (i) and (ii) hold true, and we thereby focus our attention on the latter. For each one of these requirements, we provide equivalent mathematical formulations that are amenable to control design. Regarding (i), we consider an energy function for the power system and formalize it as identifying a controller that guarantees that the time evolution of this energy function along every trajectory of the dynamics is non-decreasing. Regarding (ii), we show that this condition is equivalent to having the controller make the safe frequency interval forward invariant. To avoid discontinuities in the controller design on the boundary of the invariant set, we report to the idea of barrier functions to have the control effort gradually kick in as the state trajectory approaches the boundary. Our final step is to use the identified constraints to synthesize a specific controller that satisfies both and is distributed. The latter is a consequence of the fact that, for each bus, the constraints only involve the state of the bus and that of neighboring states. We analyze its robustness properties against measure error and parameter uncertainty, quantify its magnitude when the initial state is uncertain, and provide an estimation on the frequency convergence rate from the unsafe to the safe region for each controlled generator. Finally, we illustrate the performance and design trade-offs of the proposed controller on the IEEE 39-bus power network.

\section{Preliminaries}

In this section we introduce basic notation and notions from set invariance and graph theory.

Notation. Let $\mathbb{N}, \mathbb{R}, \mathbb{R}_{>}$, and $\mathbb{R}_{\geqslant}$denote the set of natural, real, strictly positive, and nonnegative real numbers, respectively. Variables are assumed to belong to the Euclidean space unless specified otherwise. For $a, b \in \mathbb{N}$, denote $[a, b]_{\mathbb{N}} \triangleq\{x \in \mathbb{N} \mid a \leqslant$ $x \leqslant b\}$. Given $\mathscr{C} \subset \mathbb{R}^{n}, \partial \mathscr{C}$ denotes its boundary. We let $\|\cdot\|_{2}$ denote the 2-norm on $\mathbb{R}^{n}$. For a point $x \in \mathbb{R}^{n}$ and $r \in \mathbb{R}_{>}$, denote $B_{r}(x) \triangleq\left\{x^{\prime} \in \mathbb{R}^{n} \mid\left\|x^{\prime}-x\right\|_{2} \leqslant r\right\}$. Denote $\mathbf{1}_{n}$ and $\mathbf{0}_{n}$ in $\mathbb{R}^{n}$ as the vector of all ones and zeros, respectively. For $A \in \mathbb{R}^{m \times n}$, let $[A]_{i}$ and $[A]_{i j}$ denote its $i$ th row and $(i, j)$ th element. We denote by $A^{\dagger}$ its unique Moore-Penrose pseudoinverse and by range $(A)$ its column space. A continuous function $\alpha: \mathbb{R} \rightarrow \mathbb{R}$ is of class- $\mathscr{K}$ if it is strictly increasing and $\alpha(0)=0$. Given a differentiable function $l: \mathbb{R}^{n} \rightarrow \mathbb{R}$, we let $\nabla l$ denote its gradient. A function $f: \mathbb{R}_{\geqslant} \times \mathbb{R}^{n} \rightarrow \mathbb{R}^{n},(t, x) \rightarrow f(t, x)$ is Lipschitz in $x$ (uniformly in $t$ ) if for every $x_{0} \in \mathbb{R}^{n}$, there exist $L, r>0$ such that $\| f(t, x)-$ $f(t, y)\left\|_{2} \leqslant L\right\| x-y \|_{2}$ for any $x, y \in B_{r}\left(x_{0}\right)$ and any $t \geqslant 0$.

Set invariance. We introduce here notions of forward invariance Khalil [2002]. Consider the non-autonomous system on $\mathbb{R}^{n}$,

$$
\dot{x}=f(t, x), \quad x(0)=x_{0},
$$

where $f: \mathbb{R}_{\geqslant} \times \mathbb{R}^{n} \rightarrow \mathbb{R}^{n}$. We assume $f$ is piecewise continuous in $t$ and Lipschitz in $x$, so that the solution of (1) exists and is unique. A set $\mathscr{C} \in \mathbb{R}^{n}$ is (forward) invariant for system (1) if for every initial condition $x_{0} \in \mathscr{C}$, the solution starting from $x_{0}$ satisfies $x(t) \in \mathscr{C}$ for all $t \geqslant 0$. The following result states a sufficient and necessary condition for a set to be forward invariant for (1).

Lemma 2.1 (Nagumo's Theorem Blancini and Miani [2008]). Let $l: \mathbb{R}^{n} \rightarrow \mathbb{R}$ be continuously differentiable and let $\mathscr{C} \triangleq$ $\{x \mid l(x) \leqslant 0\}$. Suppose that for all $x \in \mathscr{C}$, there exists $s \in \mathbb{R}^{n}$ such that $l(x)+\nabla l(x)^{T} s<0$. Furthermore, suppose there exists a Lipschitz function $\phi: \mathbb{R}^{n} \rightarrow \mathbb{R}^{n}$ such that $\nabla l(x)^{T} \phi(x)<0$ for all $x \in \partial \mathscr{C}$. Then $\mathscr{C}$ is forward invariant if and only if $\nabla l(x)^{T} f(t, x) \leqslant 0$ for all $x \in \partial \mathscr{C}$.

The assumptions in Nagumo's Theorem ensure that the set $\mathscr{C}$ is regular enough to have a well-defined interior and boundary.

Graph theory. We present basic notions in algebraic graph theory from Bullo et al. [2009], Biggs [1994]. An undirected graph is a pair $\mathscr{G}=(\mathscr{I}, \mathscr{E})$, where $\mathscr{I}=\{1, \ldots, n\}$ is the vertex set and $\mathscr{E}=\left\{e_{1}, \ldots, e_{m}\right\} \subseteq \mathscr{I} \times \mathscr{I}$ is the edge set. A path is an ordered sequence of vertices such that any pair of consecutive vertices in the sequence is an edge of the graph. A graph is connected if there exists a path between any two vertices. Two nodes are neighbors if there exists an edge linking them. Denote by $\mathscr{N}(i)$ the set of neighbors of node $i$. For each edge $e_{k} \in \mathscr{E}$ with vertices $i, j$, the orientation procedure consists of choosing either $i$ or $j$ to be the positive end of $e_{k}$ and the other vertex to be the negative end. The incidence matrix $D=\left(d_{k i}\right) \in \mathbb{R}^{m \times n}$ associated with $\mathscr{G}$ is then defined as

$$
d_{k i}= \begin{cases}1 & \text { if } i \text { is the positive end of } e_{k} \\ -1 & \text { if } i \text { is the negative end of } e_{k} \\ 0 & \text { otherwise }\end{cases}
$$

\section{Problem statement}

In this section we introduce the dynamical model for the power network and state our control objective.

\subsection{Power network model}

The power network is encoded by a connected undirected graph $\mathscr{G}=(\mathscr{I}, \mathscr{E})$, where $\mathscr{I}=\{1,2, \cdots, n\}$ is the collection of buses and $\mathscr{E}=\left\{e_{1}, \cdots, e_{m}\right\} \subseteq \mathscr{I} \times \mathscr{I}$ is the collection of transmission lines. For each node $i \in \mathscr{I}$, let $\theta_{i} \in \mathbb{R}, \omega_{i} \in \mathbb{R}$ and $p_{i} \in \mathbb{R}$ denote its voltage angle, shifted voltage frequency relative to the nominal frequency, and constant active power injection, respectively. We partition buses into $\mathfrak{C}$ and $\mathscr{I} \backslash \mathfrak{C}$, where every bus $i \in \mathfrak{C}$ requires an individual transient frequency regulation realized via an exogenous control command $u_{i}$. The dynamics is described by the swing equations for voltage angles and frequencies,

$$
\begin{aligned}
\dot{\theta}_{i}(t) & =\omega_{i}(t), \forall i \in \mathscr{I}, \\
M_{i} \dot{\omega}_{i}(t) & =-E_{i} \omega_{i}(t)-\sum_{j \in \mathscr{N}(i)} b_{i j} \sin \left(\theta_{i}(t)-\theta_{j}(t)\right)+u_{i}(t)+p_{i}, \forall i \in \mathfrak{C}, \\
M_{i} \dot{\omega}_{i}(t) & =-E_{i} \omega_{i}(t)-\sum_{j \in \mathscr{N}(i)} b_{i j} \sin \left(\theta_{i}(t)-\theta_{j}(t)\right)+p_{i}, \forall i \in \mathscr{I} \backslash \mathfrak{C},
\end{aligned}
$$

where $b_{i j} \in \mathbb{R}_{>}$is the susceptance of the line connecting bus $i$ and $j$, and $M_{i} \in \mathbb{R}_{\geqslant}$and $E_{i} \in \mathbb{R}_{\geqslant}$are the inertia and damping coefficients of bus $i \in \mathscr{I}$. For simplicity, we assume that they are all strictly positive.

For our purposes, it is convenient to rewrite the dynamics (2) in a more compact way. Let $\theta \triangleq\left[\theta_{1}, \cdots, \theta_{n}\right]^{T} \in \mathbb{R}^{n}$, $\omega \triangleq\left[\omega_{1}, \cdots, \theta_{n}\right]^{T} \in \mathbb{R}^{n}$ and $p \triangleq\left[p_{1}, \cdots, p_{n}\right]^{T} \in \mathbb{R}^{n}$ be the collection of voltage angles, frequencies, and power injections. Let $D \in \mathbb{R}^{m \times n}$ be the incidence matrix corresponding to an arbitrary graph orientation, and define the voltage angle difference vector

$$
\lambda \triangleq D \theta \in \mathbb{R}^{m}
$$


Denote by $Y_{b} \in \mathbb{R}^{m \times m}$ the diagonal matrix whose $k$ th diagonal item represents the susceptance of the transmission line $e_{k}$ connecting bus $i$ and $j$, i.e., $\left[Y_{b}\right]_{k, k}=b_{i j}$, for $k=1,2, \cdots, m$. We rewrite the dynamics (2) in terms of $\lambda$ and $\omega$ as

$$
\begin{aligned}
\dot{\lambda}(t) & =D \omega(t), \\
M_{i} \dot{\omega}_{i}(t) & =-E_{i} \omega_{i}(t)-\left[D^{T} Y_{b}\right]_{i} \sin \lambda(t)+u_{i}(t)+p_{i}, \forall i \in \mathfrak{C}, \\
M_{i} \dot{\omega}_{i}(t) & =-E_{i} \omega_{i}(t)-\left[D^{T} Y_{b}\right]_{i} \sin \lambda(t)+p_{i}, \forall i \in \mathscr{I} \backslash \mathfrak{C},
\end{aligned}
$$

where $\sin \lambda(t) \in \mathbb{R}^{m}$ is the component-wise sine value of $\lambda(t)$. Note that the transformation (3) enforces $\lambda(0) \in \operatorname{range}(D)$. We refer to an initial condition satisfying this equation as admissible. When convenient, for conciseness, we use $x(t) \triangleq(\lambda(t), \omega(t)) \in$ $\mathbb{R}^{m+n}$ to denote the collection of all states, and we neglect its dependence on $t$ if the context is clear.

The trajectories $(\lambda(t), \omega(t))$ locally converge to a unique equilibrium point if all $u_{i}$ 's are set to zero. Specifically, let $L \triangleq$ $D^{T} Y_{b} D$ and $L^{\dagger}$ be its pseudoinverse. Define $\omega^{\infty} \triangleq \frac{\sum_{i=1}^{n} p_{i}}{\sum_{i=1}^{n} E_{i}}, E \triangleq$ $\operatorname{diag}\left(E_{1}, E_{2}, \cdots, E_{n}\right)$, and $\tilde{p} \triangleq p-\omega^{\infty} E \mathbf{1}_{n}$. If

$$
\left\|L^{\dagger} \tilde{p}\right\|_{\mathscr{E}, \infty}<1
$$

where $\|y\|_{\mathscr{E}, \infty} \triangleq \max _{(i, j) \in \mathscr{E}}\left|y_{i}-y_{j}\right|$, then there exists $\lambda^{\infty} \in \Gamma \triangleq$ $\left\{\lambda|| \lambda_{i} \mid<\pi / 2\right\}$ unique in $\Gamma_{\mathrm{cl}} \triangleq\left\{\lambda|| \lambda_{i} \mid \leqslant \pi / 2\right\}$ such that

$$
\tilde{p}=D^{T} Y_{b} \sin \lambda^{\infty} \text { and } \lambda^{\infty} \in \operatorname{range}(D) \text {. }
$$

According to |Dörfler et al. 2013, Lemma 2 and inequality (S17)], system (4) with $u_{i} \equiv 0$ for every $i \in \mathfrak{C},\left(\lambda^{\infty}, \omega^{\infty} \mathbf{1}_{n}\right)$ is stable. Furthermore, $(\lambda(t), \omega(t))$ locally converges to $\left(\lambda^{\infty}, \omega^{\infty} \mathbf{1}_{n}\right)$ provided $\lambda(0) \in \operatorname{range}(D)$. Throughout the rest of the paper, we assume that condition (5) holds.

\subsection{Control goal}

Our goal is to design a state-feedback controller for each bus $i \in \mathfrak{C}$ that guarantees that the frequency transient behavior stays within desired safety bounds while, at the same time, preserving the stability properties that the system (4) enjoys when no external input $u_{i}$ is present. We state these requirements explicitly next.

Stability and convergence requirement: Since the system (4) without $u_{i}$ is locally stable, we require that the same system with the proposed controller $u_{i}$ is also locally stable. Furthermore, for every admissible initial condition, the two systems should converge to the same equilibrium $\left(\lambda^{\infty}, \omega^{\infty} \mathbf{1}_{n}\right)$, meaning that $u_{i}$ only affects the transient behavior.

Frequency invariance requirement: For each $i \in \mathfrak{C}$, let $\underline{\omega}_{i} \in \mathbb{R}$ and $\bar{\omega}_{i} \in \mathbb{R}$ be lower and upper safe frequency bounds, where $\underline{\omega}_{i}<\bar{\omega}_{i}$. We require that the frequency $\omega_{i}(t)$ stays inside the safe region $\left[\underline{\omega}_{i}, \bar{\omega}_{i}\right]$ for any $t>0$, provided that the initial frequency $\omega_{i}(0)$ lies inside $\left[\underline{\omega}_{i}, \bar{\omega}_{i}\right]$. This forward invariance requirement corresponds to underfrequency/overfrequency avoidance.

Attractivity requirement: If, for some $i \in \mathfrak{C}$, the initial frequency $\omega_{i}(0) \notin\left[\underline{\omega}_{i}, \bar{\omega}_{i}\right]$, then after a finite time, $\omega_{i}$ enters the safe region and never leaves afterwards. This requirement corresponds to underfrequency/overfrequency recovery.

In addition to these requirements, we also seek the designed controller to be Lipschitz as a function of the state. This guarantees the existence and uniqueness of solutions for the closed-loop system and, at the same time, provides robustness for practical implementation against errors in state measurements.

Remark 3.1 (Selection of buses with transient frequency specification). The set $\mathfrak{C}$ consists of buses belonging to either of the following two types: a) buses with specified over/underfrequency requirement [Pouyan et al., 2006] and b) buses whose transient frequency behavior is key in evaluating system performance, or are used as indexes for load shedding schemes [Miller et al. 2011]. We assume each individual bus in $\mathfrak{C}$ is equipped with an external input directly tuning its transient behavior. We show later that this is necessary condition to obtain frequency invariance guarantees.

Note that the attractivity requirement is automatically satisfied once the controller meets the first two requirements, provided that $\omega^{\infty} \in\left(\underline{\omega}_{i}, \bar{\omega}_{i}\right)$. However, in general it is still of interest to provide estimates for how fast the frequency reaches the safe region. Our objective is to design a controller that satisfies the above three requirements simultaneously and is distributed, in the sense that each bus can implement it using its own information and that of its neighboring buses and transmission lines.

\section{Constraints on controller design}

In this section, we identify constraints on the controller design that provide sufficient conditions to ensure, on the one hand, the stability and convergence requirement and, on the other hand, the frequency invariance requirement.

\subsection{Constraint ensuring stability and convergence}

We establish a stability constraint by identifying an energy function and restricting the input so that its evolution along every trajectory of the closed-loop dynamics is monotonically nonincreasing. We select the following energy function [ $\mathrm{Vu}$ et al. 2017.

$$
V(\lambda, \omega) \triangleq \frac{1}{2} \sum_{i=1}^{n} M_{i}\left(\omega_{i}-\omega^{\infty}\right)^{2}+\sum_{j=1}^{m}\left[Y_{b}\right]_{j, j} a\left(\lambda_{j}\right)
$$

where $a\left(\lambda_{j}\right) \triangleq \cos \lambda_{j}^{\infty}-\cos \lambda_{j}-\lambda_{j} \sin \lambda_{j}^{\infty}+\lambda_{j}^{\infty} \sin \lambda_{j}^{\infty}$. The next result uses the LaSalle Invariance Principle to show this property.

Lemma 4.1 (Sufficient condition for local stability and convergence). Consider the system (4). Under condition (5), further suppose that, for every $i \in \mathfrak{C}, u_{i}: \mathbb{R}^{m+n} \times \mathbb{R}^{n} \rightarrow \mathbb{R},(x, y) \mapsto u_{i}(x, y)$ is Lipschitz in $x$. Let $c \triangleq \min _{\lambda \in \partial \Gamma_{\mathrm{cl}}} V\left(\lambda, \omega^{\infty} \mathbf{1}_{n}\right)$ and define

$$
\Phi \triangleq\left\{(\lambda, \omega) \mid \lambda \in \Gamma_{\mathrm{cl}}, V(\lambda, \omega) \leqslant c / \beta\right\}
$$

with $\beta \in \mathbb{R}_{>}$. If for every $i \in \mathfrak{C}, x \in \mathbb{R}^{m+n}$, and $p \in \mathbb{R}^{n}$,

$$
\begin{aligned}
\left(\omega_{i}-\omega^{\infty}\right) u_{i}(x, p) \leqslant 0 & \text { if } \omega_{i} \neq \omega^{\infty} \\
u_{i}(x, p) & =0 \quad \text { if } \omega_{i}=\omega^{\infty}
\end{aligned}
$$

then the following results hold provided $\lambda(0) \in \operatorname{range}(D)$ and $(\lambda(0), \omega(0)) \in \Phi$ for some $\beta>1$ :

(i) The solution of the closed-loop system exists and is unique for any $t \geqslant 0$;

(ii) $\lambda(t) \in \operatorname{range}(D)$ and $(\lambda(t), \omega(t)) \in \Phi$ for any $t \geqslant 0$;

(iii) $\left(\lambda^{\infty}, \omega^{\infty} \mathbf{1}_{n}\right)$ is stable, and $(\lambda(t), \omega(t)) \rightarrow\left(\lambda^{\infty}, \omega^{\infty} \mathbf{1}_{n}\right)$ as $t \rightarrow$ $\infty$. 
PROOF. To prove $(i)$, as $(x, y) \mapsto u_{i}(x, y)$ is Lipschitz in $x$, there exists a unique local solution over $[0, \delta]$ for some $\delta>0$, according to [Khalil, 2002, Theorem 3.1]. Let $[0, T)$ be the maximal interval of existence. We then show that $\Phi$ is non-empty and compact, and that $(\lambda(t), \omega(t))$ lies entirely in $\Phi$ for any $t \in[0, T)$. These two facts together, by [Khali1, 2002, Theorem 3.3], imply the existence and uniqueness of the solution for every $t \geqslant 0$. To show the non-emptiness of $\Phi$, note that in (7) if $\left|\lambda_{i}\right| \leqslant \pi / 2$ and $\left|\lambda_{i}^{\infty}\right|<\pi / 2$, then $a\left(\lambda_{i}\right) \geqslant 0$, which implies that $V(\lambda, \omega) \geqslant 0$ for every $\lambda \in \Gamma_{\mathrm{cl}}$ and every $\omega \in \mathbb{R}^{n}$; hence $c \geqslant 0$. Then $\left(\lambda^{\infty}, \omega^{\infty} \mathbf{1}_{n}\right) \in \Phi$ as $V\left(\lambda^{\infty}, \omega^{\infty} \mathbf{1}_{n}\right)=0$. To show the compactness of $\Phi$, note that the set is clearly closed. Since the polytope $\Gamma_{\mathrm{cl}}$ is bounded, the variable $\lambda$ is bounded too. Therefore, $a\left(\lambda_{i}\right)$ is bounded for every $i \in[1, m]_{\mathbb{N}}$. Since $V(\lambda, \omega) \leq c / \beta$, we deduce that $\sum_{i=1}^{n} M_{i}\left(\omega_{i}-\omega^{\infty}\right)^{2}$ is bounded, implying that $\omega$ is bounded. Hence, $\Phi$ is bounded.

Regarding statement (ii), note that $\lambda(t) \in \operatorname{range}(D)$ holds for every $t \geqslant 0$ since both $\lambda(0)$ and $\dot{\lambda}(t)$ lie in range $(D)$. To establish the invariance of $\Phi$, we examine the evolution of the function $V$ along the dynamics (4),

$$
\begin{aligned}
\dot{V}(\lambda, \omega) & =\sum_{i=1}^{n}\left(\omega_{i}-\omega^{\infty}\right)\left(-E_{i} \omega_{i}-\left[D^{T} Y_{b}\right]_{i} \sin \lambda+p_{i}\right) \\
& +\sum_{i \in \mathfrak{C}}\left(\omega_{i}-\omega^{\infty}\right) u_{i}(x, p)+\sum_{j=1}^{m}\left[Y_{b}\right]_{j, j}\left(\sin \lambda_{j}-\sin \lambda_{j}^{\infty}\right)[D]_{j} \omega \\
& =-\sum_{i=1}^{n} E_{i}\left(\omega_{i}-\omega^{\infty}\right)^{2}+\sum_{i \in \mathfrak{C}}\left(\omega_{i}-\omega^{\infty}\right) u_{i}(x, p) \\
& \leqslant-\sum_{i=1}^{m} E_{i}\left(\omega_{i}-\omega^{\infty}\right)^{2} \leqslant 0,
\end{aligned}
$$

where we have employed (10) in the second equality. This monotonicity of $V$ implies that the constraint $V(\lambda, \omega) \leqslant c / \beta$ defining $\Phi$ can never be violated. Now if there exists a time $t_{1}>0$ such that $\left(\lambda\left(t_{1}\right), \omega\left(t_{1}\right)\right) \notin \Phi$, then it must be the case where $\lambda\left(t_{1}\right) \notin \Gamma$. By the continuity of the trajectory, there must exist another time $t_{2}$ before $t_{1}$ such that $\lambda\left(t_{2}\right) \in \partial \Gamma_{\mathrm{cl}}$, in which case $V\left(\lambda\left(t_{2}\right), \omega\left(t_{2}\right)\right) \geqslant V\left(\lambda\left(t_{2}\right), \omega^{\infty} \mathbf{1}_{n}\right) \geqslant c>c / \beta$, which is a contradiction. Hence $\Phi$ is invariant.

To prove (iii), notice that, for any $(\lambda, \omega) \in \Phi, \dot{V}(\lambda, \omega) \leqslant 0$; second, $V\left(\lambda^{\infty}, \omega^{\infty} \mathbf{1}_{n}\right)=0$; third, $V(\lambda, \omega)>0$, for every $(\lambda, \omega) \in$ $\Phi$ with $(\lambda, \omega) \neq\left(\lambda^{\infty}, \omega^{\infty} \mathbf{1}_{n}\right)$. By [Khalil, 2002, Theorem 4.1], $\left(\lambda^{\infty}, \omega^{\infty} \mathbf{1}_{n}\right)$ is stable. Finally, to establish convergence, let

$$
\Omega \triangleq \Phi \cap\{(\lambda, \omega) \mid \lambda \in \operatorname{range}(D)\}
$$

Note that $(\lambda(0), \omega(0)) \in \Omega$. Clearly, the set $\Omega$ is compact and invariant with respect to the dynamics (4a)-(4c) with controller satisfying (9). Noticing that $\dot{V}(\lambda, \omega)=0$ implies $\omega=\omega^{\infty} \mathbf{1}_{n}$, let $S \triangleq\left\{(\lambda, \omega) \mid \omega=\omega^{\infty} \mathbf{1}_{n}\right\} \cap \Omega$. It is easy to see that no solution can identically stay in $S$ other than the trivial solution $(\lambda(t), \omega(t)) \equiv\left(\lambda^{\infty}, \omega^{\infty} \mathbf{1}_{n}\right)$. The conclusion then follows from the LaSalle Invariance Principle [Khalil, 2002, Theorem 4.4].

Remark 4.2 (Computation of the region of attraction). The set $\Phi$ is an estimate of the region of attraction but its explicit computation requires the solution of a non-convex optimization problem to determine the value of $c$. We can equivalently compute $c$ by solving $2 m$ convex problems. For each $j \in[1, m]_{\mathbb{N}}$, let

$$
\bar{c}_{j} \triangleq \min _{\substack{\lambda_{j}=\pi / 2 \\\left|\lambda_{i}\right| \leqslant \pi / 2, \forall i \neq j}} V\left(\lambda, \omega^{\infty} \mathbf{1}_{n}\right), \quad \underline{c}_{j} \triangleq \min _{\substack{\lambda_{j}=-\pi / 2 \\\left|\lambda_{i}\right| \leqslant \pi / 2, \forall i \neq j}} V\left(\lambda, \omega^{\infty} \mathbf{1}_{n}\right)
$$

Note that these problems are convex, as the Hessian of $V\left(\tilde{\lambda}, \omega^{\infty} \mathbf{1}_{n}\right)$ with respect to $\tilde{\lambda}, \nabla^{2} V=\operatorname{diag}\left(\left[Y_{b}\right]_{1,1} \cos \left(\lambda_{1}\right), \cdots\right.$, $\left.\left[Y_{b}\right]_{m, m} \cos \left(\lambda_{m}\right)\right)$, is positive definite on $\Gamma_{\mathrm{cl}}$, and the feasible set is a closed convex subset of $\Gamma_{\mathrm{cl}}$. One can easily see that $c=\min _{j \in[1, m]_{\mathbb{N}}}\left\{\bar{c}_{j}, \underline{c}_{j}\right\}$.

On the other hand, although it is easy to check if a given initial state belongs to $\Phi$, it is difficult to characterize its geometric shape. The work [Vu et al. 2018] shows that, for suitable $\bar{c}>0$ determined via a convex quadratic program, the ellipsoid

$$
\bar{\Phi} \triangleq\{(\lambda, \omega) \mid \bar{V}(\omega, \lambda) \leqslant \bar{c}\}
$$

is a subset of $\Phi$ (here $\bar{V}(\omega, \lambda) \triangleq \frac{1}{2} \sum_{i=1}^{n} M_{i}\left(\omega_{i}-\omega^{\infty}\right)^{2}+$ $\frac{1}{2} \sum_{j=1}^{m}\left[Y_{b}\right]_{j, j}\left(\lambda_{j}-\lambda_{j}^{\infty}\right)^{2}$ is quadratic). Lemma 4.1 remains valid if $\Phi$ is replaced by $\bar{\Phi}$.

\subsection{Constraint ensuring frequency invariance}

We next focus our attention on the frequency invariance requirement. We start by defining the invariant sets we are interested in,

$$
\overline{\mathscr{C}}_{i} \triangleq\left\{x \mid \omega_{i}-\bar{\omega}_{i} \leqslant 0\right\}, \quad \underline{\mathscr{C}}_{i} \triangleq\left\{x \mid \underline{\omega}_{i}-\omega_{i} \leqslant 0\right\} .
$$

The characterization stated in the next result directly follows from Nagumo's Theorem.

Lemma 4.3 (Sufficient and necessary condition for frequency invariance). Assume that the solution of (4) exists and is unique for every admissible initial condition. Then, for any $i \in \mathfrak{C}$, the sets $\overline{\mathscr{C}}_{i}$ and $\underline{\mathscr{C}}_{i}$ are invariant if and only if for every $x \in \mathbb{R}^{m+n}$ and $p \in \mathbb{R}^{n}$,

$$
\begin{aligned}
u_{i}(x, p)-q_{i}(x, p) \leqslant 0 & \text { if } \omega_{i}=\bar{\omega}_{i}, \\
-u_{i}(x, p)+q_{i}(x, p) \leqslant 0 & \text { if } \omega_{i}=\underline{\omega}_{i},
\end{aligned}
$$

where $q_{i}(x, p) \triangleq E_{i} \omega_{i}+\left[D^{T} Y_{b}\right]_{i} \sin \lambda-p_{i}$.

PROOF. For simplicity, we only deal with the case of $\overline{\mathscr{C}}_{i}$ (the other case follows similarly). For each $i \in \mathfrak{C}$, let $\bar{l}_{i}, \underline{l}_{i}: \mathbb{R}^{n} \rightarrow \mathbb{R}$ be defined by $\bar{l}_{i}(x) \triangleq \omega_{i}-\bar{\omega}_{i}$ and $\underline{l}_{i}(x) \triangleq-\omega_{i}+\underline{\omega}_{i}$. Notice that, by letting $s=-\mathbf{1}_{m+n}$ and $\phi(x) \equiv-\mathbf{1}_{m+n}$, one has that $\bar{l}_{i}(x)+$ $\nabla \bar{l}_{i}(x)^{T} s<0$ for every $x \in \overline{\mathscr{C}}_{i}$ and $\nabla \bar{l}_{i}(x)^{T} \phi(x)<0$ for every $x \in \partial \overline{\mathscr{C}}_{i}$, and hence the assumptions in Nagumo's Theorem hold. Denote by $f(t, x)$ the right-hand side of the dynamics (4). Then $\overline{\mathscr{C}}_{i}$ is invariant if and only if $\nabla \bar{l}_{i}(x)^{T} f(t, x) \leqslant 0$ when $\omega_{i}(t)=\bar{\omega}_{i}$, which is equivalent to (13a).

From Lemma 4.3, one sees that if some bus $j \in \mathfrak{C}$ does not possess an external control input (i.e., $u_{j} \equiv 0$ ), then one can not guarantee the invariance of $\overline{\mathscr{C}}_{j}$ and $\underline{\mathscr{C}}_{j}$, since without an active control signal, condition (13) can easily be violated. The characterization of Lemma 4.3 points to the value of the input at the boundary of $\overline{\mathscr{C}}_{i}$ and $\underline{\mathscr{C}}_{i}$. However, having a controller that is only nonvanishing at such points is undesirable, as the actuator effort would be discontinuous, affecting the system evolution. A more sensible 


$$
\begin{aligned}
& \sum_{i=1}^{n}\left(\omega_{i}-\omega^{\infty}\right)\left(-\left[D^{T} Y_{b}\right]_{i} \sin \lambda+p_{i}-\omega^{\infty} E_{i}\right)+\sum_{j=1}^{m}\left[Y_{b}\right]_{j, j}\left(\sin \lambda_{j}-\sin \lambda_{j}^{\infty}\right)[D]_{j} \omega \\
= & \sum_{i=1}^{n}\left(\omega_{i}-\omega^{\infty}\right)\left(-\left[D^{T} Y_{b}\right]_{i} \sin \lambda+p_{i}-\omega^{\infty} E_{i}\right)+\sum_{j=1}^{m}\left(\sin \lambda_{j}-\sin \lambda_{j}^{\infty}\right)\left[Y_{b} D\right]_{j}\left(\omega-\omega^{\infty} \mathbf{1}_{n}\right) \\
= & \sum_{i=1}^{n}\left(\omega_{i}-\omega^{\infty}\right)\left(p_{i}-\omega^{\infty} E_{i}\right)-\sum_{j=1}^{m}\left(\sin \lambda_{j}^{\infty}\right)\left[Y_{b} D\right]_{j}\left(\omega-\omega^{\infty} \mathbf{1}_{n}\right) \\
= & \sum_{i=1}^{n}\left(\omega_{i}-\omega^{\infty}\right)\left(p_{i}-\omega^{\infty} E_{i}-D^{T} Y_{b} \sin \lambda_{i}^{\infty}\right)=\left(\omega-\omega^{\infty} \mathbf{1}_{n}\right)^{T}\left(\tilde{p}-D^{T} Y_{b} \sin \lambda^{\infty}\right)=0 .
\end{aligned}
$$

policy is to have the controller become active as the system state gets closer to the boundary of these sets, and do so in a gradual way. This is captured by the following result.

Lemma 4.4 (Sufficient condition for frequency invariance). Assume that the solution of (4) exists and is unique for every admissible initial condition. For each $i \in \mathfrak{C}$, let $\bar{\omega}_{i}^{\text {th }}, \underline{\omega}_{i}^{\text {th }} \in \mathbb{R}$ be such that $\underline{\omega}_{i}<\underline{\omega}_{i}^{\text {th }}<\bar{\omega}_{i}^{\text {th }}<\bar{\omega}_{i}$ and let $\bar{\alpha}_{i}$ and $\underline{\alpha}_{i}$ be functions of class- $\mathscr{K}$. If for every $x \in \mathbb{R}^{m+n}$ and $p \in \mathbb{R}^{n}$,

$$
\left(\omega_{i}-\bar{\omega}_{i}^{\mathrm{th}}\right)\left(u_{i}(x, p)-q_{i}(x, p)\right) \leqslant-\bar{\alpha}_{i}\left(\omega_{i}-\bar{\omega}_{i}\right)
$$

if $\bar{\omega}_{i}^{\mathrm{th}}<\omega_{i} \leqslant \bar{\omega}_{i}$, and

$$
\left(\underline{\omega}_{i}^{\mathrm{th}}-\omega_{i}\right)\left(-u_{i}(x, p)+q_{i}(x, p)\right) \leqslant-\underline{\alpha}_{i}\left(\underline{\omega}_{i}-\omega_{i}\right)
$$

if $\underline{\omega}_{i} \leqslant \omega_{i}<\underline{\omega}_{i}^{\mathrm{th}}$, then $\overline{\mathscr{C}}_{i}$ and $\underline{\mathscr{C}}_{i}$ are invariant.

The proof of Lemma 4.4 follows by noting that, when $\omega_{i}=\bar{\omega}_{i}$ (resp. $\omega_{i}=\underline{\omega}_{i}$ ), condition (14a) (resp. (14b)) becomes (13a) (resp. (13b)). The introduction of class- $\mathscr{K}$ functions enables the design of controllers that gradually kick in as the margin for satisfying the requirement for frequency invariance gets increasingly small. In fact, using (4), we can equivalently write (14a) as

$$
M \dot{\omega}_{i} \leqslant-\bar{\alpha}_{i}\left(\omega_{i}-\bar{\omega}_{i}\right) /\left(\omega_{i}-\bar{\omega}_{i}^{\text {th }}\right), \quad \text { if } \bar{\omega}_{i}^{\text {th }}<\omega_{i} \leqslant \bar{\omega}_{i}
$$

Notice that, as $\omega_{i}$ grows from the threshold $\bar{\omega}_{i}^{\text {th }}$ to the safe bound $\bar{\omega}_{i}$, the value of $-\bar{\alpha}_{i}\left(\omega_{i}-\bar{\omega}_{i}\right) /\left(\omega_{i}-\bar{\omega}_{i}^{\text {th }}\right)$ monotonically decreases to 0 . Thus, the constraint on $\dot{\omega}_{i}$ becomes tighter (while allowing $\dot{\omega}_{i}$ to still be positive) as $\omega_{i}$ approaches $\bar{\omega}_{i}$, and when $\omega_{i}$ hits $\bar{\omega}_{i}$, prescribes $\dot{\omega}_{i}$ to be nonpositive to ensure invariance. It is interesting to point out the trade-offs present in the choice of class- $\mathscr{K}$ functions. A function with a large derivative, for instance, corresponds to a controller design that allows the derivative above to be significant near the boundary, at the risk of increasing the sensitivity to changes in the state. We re-examine this point later after introducing our specific controller design.

\section{Distributed controller synthesis}

In this section we introduce a distributed controller design that meets the stability and convergence condition (9) as well as the frequency invariance condition (14). Our next result formally introduces this controller and characterizes its continuity property.
Proposition 5.1 (Distributed frequency controller). For each $i \in$ $\mathfrak{C}$, let $\bar{\alpha}_{i}$ and $\underline{\alpha}_{i}$ be Lipschitz functions of class- $\mathscr{K}$. Then,

$$
u_{i}(x, p)= \begin{cases}\min \left\{0, \frac{-\bar{\alpha}_{i}\left(\omega_{i}-\bar{\omega}_{i}\right)}{\omega_{i}-\bar{\omega}_{i}^{\mathrm{th}}}+q_{i}(x, p)\right\} & \omega_{i}>\bar{\omega}_{i}^{\mathrm{th}}, \\ 0 & \underline{\omega}_{i}^{\mathrm{th}} \leqslant \omega_{i} \leqslant \bar{\omega}_{i}^{\mathrm{th}}, \\ \max \left\{0, \frac{\underline{\alpha}_{i}\left(\underline{\omega}_{i}-\omega_{i}\right)}{\underline{\omega}_{i}^{\mathrm{th}}-\omega_{i}}+q_{i}(x, p)\right\} & \omega_{i}<\underline{\omega}_{i}^{\mathrm{th}},\end{cases}
$$

is Lipschitz in its first argument.

PROOF. Let $i \in \mathfrak{C}$. We show that for any $x \in \mathbb{R}^{m+n}$, there exist $L, r \in \mathbb{R}_{>}$such that $\left|u_{i}(y, p)-u_{i}(z, p)\right| \leqslant L\|y-z\|$ for any $y, z \in$ $B_{r}(x)$. Notice that this condition holds true for $x$ belonging to $\mathbb{H} \triangleq\left\{x \in \mathbb{R}^{m+n} \mid \omega_{i} \neq \bar{\omega}_{i}^{\text {th }}, \omega_{i} \neq \underline{\omega}_{i}^{\text {th }}\right\}$, in that $x \mapsto \frac{\left.-\bar{\alpha}_{i}\left(\omega_{i}-\bar{\omega}_{i}\right)\right)}{\left(\omega_{i}-\bar{\omega}_{i}^{\text {th }}\right)}+$ $q_{i}(x, p)$ (resp. $\left.x \mapsto \frac{\underline{\alpha}_{i}\left(\underline{\omega}_{i}-\omega_{i}\right)}{\underline{\omega}_{i}^{\text {th }}-\omega_{i}}+q_{i}(x, p)\right)$ is Lipschitz for any $x$ in $\mathbb{H}$, and the min (resp. $\max$ )) operator preserves Lipschitz continuity. Hence we only need to establish Lipschitzness for $x \notin \mathbb{H}$. For simplicity we only reason for the case when $x$ satisfies $\omega_{i}=\bar{\omega}_{i}^{\text {th }}$. Denote $r_{0} \triangleq \min \left\{\frac{1}{2}\left(\bar{\omega}_{i}-\bar{\omega}_{i}^{\text {th }}\right), \frac{1}{2}\left(\bar{\omega}_{i}^{\text {th }}-\underline{\omega}_{i}^{\text {th }}\right)\right\} \in \mathbb{R}_{>}$. One can see that for any $x^{\prime} \in B_{r_{0}}(x)$, it holds that $\underline{\omega}_{i}^{\text {th }} \leqslant \omega_{i}$. Next we show that there always exists $r \leqslant r_{0}$ such that

$$
\frac{-\bar{\alpha}_{i}\left(\omega_{i}-\bar{\omega}_{i}\right)}{\left(\omega_{i}-\bar{\omega}_{i}^{\text {th }}\right)}+q_{i}\left(x^{\prime}, p\right)>0
$$

for all $x^{\prime} \in B_{r}(x) \cap\left\{x^{\prime} \mid \omega_{i}>\bar{\omega}_{i}^{\text {th }}\right\}$. Notice that for any $x^{\prime} \in B_{r}(x), \omega_{i}-\bar{\omega}_{i} \leqslant \bar{\omega}_{i}^{\text {th }}+r-\bar{\omega}_{i} \leqslant \omega_{i}^{\text {th }}+\left(\bar{\omega}_{i}-\bar{\omega}_{i}^{\text {th }}\right) / 2-\bar{\omega}_{i}=$ $-\left(\bar{\omega}_{i}-\bar{\omega}_{i}^{\text {th }}\right) / 2<0$, and $q_{i}\left(x^{\prime}, p\right)=\omega_{i}+\left[D^{T}\right]_{i} \lambda-p_{i} \geqslant-(n+$ 1) $\left\|x^{\prime}\right\|_{2}-\left|p_{i}\right|$. Therefore, it holds that

$$
\frac{-\bar{\alpha}_{i}\left(\omega_{i}-\bar{\omega}_{i}\right)}{\left(\omega_{i}-\bar{\omega}_{i}^{\mathrm{th}}\right)}+q_{i}\left(x^{\prime}, p\right) \geqslant \frac{-\bar{\alpha}_{i}\left(\omega_{i}-\bar{\omega}_{i}\right)}{2 r}-(n+1)\left\|x^{\prime}\right\|_{2}-\left|p_{i}\right|
$$

It is easy to see that for any $x^{\prime} \in B_{r}(x) \cap\left\{x^{\prime} \mid \omega_{i}>\bar{\omega}_{i}^{\text {th }}\right\}$, the first term can be arbitrarily large by reducing $r$, while the other two terms are bounded; therefore, there exits $r>0$ small enough such that (17) holds. By (16), this implies that $u_{i}\left(x^{\prime}, p\right)=0$ for any $x^{\prime} \in B_{r}(x)$, and hence $u_{i}$ is Lipschitz in $x$.

Remark 5.2 (Distributed character and practical implementation). The controller (16) is distributed since each controlled bus $i \in \mathfrak{C}, u_{i}$ only utilizes $\omega_{i}, p_{i}$, and information of buses it is connected to in the power network in order to compute $\left[D^{T} Y_{b}\right]_{i} \lambda$. This term corresponds to the aggregate power flow injected at 
node $i$ from its neighboring nodes. In turn, this means that, instead of measuring $\lambda_{j}$ and its corresponding susceptance for every $i$ 's neighboring node $j$, in practice, each node can simply measure the signed power flows in each neighboring transmission lines of node $i$ and sum it up, which is equivalent to $\left[D^{T} Y_{b}\right]_{i} \lambda$ as well. $\bullet$

The next result shows that the proposed distributed controller achieves the objectives identified in Section 3 regarding stability, convergence, and frequency invariance.

Theorem 5.3 (Transient frequency control with stability guarantees). Under condition (5), let $\omega^{\infty} \in\left(\omega_{i}^{\text {th }}, \bar{\omega}_{i}^{\text {th }}\right)$ and consider the closed-loop system (4) with controller (16). If $\lambda(0) \in \operatorname{range}(D)$ and $(\lambda(0), \omega(0)) \in \Phi$ for some $\beta>1$, then

(i) The solution exists and is unique for every $t \geqslant 0$;

(ii) $\lambda(t) \in \operatorname{range}(D)$ and $(\lambda(t), \omega(t)) \in \Phi$ for any $t \geqslant 0$;

(iii) $\left(\lambda^{\infty}, \omega^{\infty} \mathbf{1}_{n}\right)$ is stable, and $(\lambda(t), \omega(t)) \rightarrow\left(\lambda^{\infty}, \omega^{\infty} \mathbf{1}_{n}\right)$ as $t \rightarrow$ $\infty$;

(iv) The controllers become inactive in finite time, i.e., there exists a time $t_{0}>0$ such that $u_{i}(x(t), p)=0$ for all $t \geqslant t_{0}$ and all $i \in \mathfrak{C}$.

(v) For any $i \in \mathfrak{C}$, if $\omega_{i}(0) \in\left[\underline{\omega}_{i}, \bar{\omega}_{i}\right]$, then $\omega_{i}(t) \in\left[\underline{\omega}_{i}, \bar{\omega}_{i}\right]$ for all $t>0$;

(vi) For any $i \in \mathfrak{C}$, if $\omega_{i}(0) \notin\left[\underline{\omega}_{i}, \bar{\omega}_{i}\right]$, then $\omega_{i}(t)$ monotonically approaches $\left[\underline{\omega}_{i}, \bar{\omega}_{i}\right]$. Furthermore, there exists a finite time $t_{1}>0$ such that $\omega_{i}(t) \in\left[\underline{\omega}_{i}, \bar{\omega}_{i}\right]$ for all $t \geqslant t_{1}$.

In addition, if $(i)$ holds for $(\lambda(0), \omega(0)) \notin \Phi$, then $(v)$ and the monotonic convergence in (vi) still hold, but with no guarantee on the existence of a finite $t_{1}$.

PROOF. It is easy to see that (16) guarantees $u_{i}(x, p) \leqslant 0$ if $\omega_{i}>$ $\bar{\omega}_{i}^{\text {th }}, u_{i}(x, p)=0$ if $\omega_{i} \in\left(\underline{\omega}_{i}^{\text {th }}, \bar{\omega}_{i}^{\text {th }}\right)$, and $u_{i}(x, p) \geqslant 0$ if $\omega_{i}<\underline{\omega}_{i}^{\text {th }}$. Therefore, (9) holds as $\omega^{\infty} \in\left(\underline{\omega}_{i}^{\text {th }}, \bar{\omega}_{i}^{\text {th }}\right)$. Hence $(i)$-(iii) directly follow from Lemma 4.1 (Proposition 5.1 justifies the Lipschitzness of the controller).

To prove (iv), we use the convergence established in (iii). For $\varepsilon=\min _{i \in \mathfrak{C}}\left\{\bar{\omega}_{i}^{\text {th }}-\omega^{\infty}, \omega^{\infty}-\underline{\omega}_{i}^{\text {th }}\right\}$, there exists $t_{0} \in \mathbb{R}>$ such that $\left\|(\lambda(t), \omega(t))-\left(\lambda^{\infty}, \omega^{\infty} \mathbf{1}_{n}\right)\right\|_{2}<\varepsilon$, for $t \geqslant t_{0}$. Therefore, for any $i \in \mathfrak{C},\left|\omega_{i}(t)-\omega^{\infty}\right| \leqslant\left\|(\lambda(t), \omega(t))-\left(\lambda^{\infty}, \omega^{\infty} \mathbf{1}_{n}\right)\right\|_{2} \leqslant \min \left\{\bar{\omega}_{i}^{\text {th }}-\right.$ $\left.\omega^{\infty}, \omega^{\infty}-\underline{\omega}_{i}^{\text {th }}\right\}$, for $t \geqslant t_{0}$, which implies $\underline{\omega}_{i}^{\text {th }} \leqslant \omega_{i}(t) \leqslant \bar{\omega}_{i}^{\text {th }}$, for $t \geqslant t_{0}$. The result follows now from the definition (16) of the controller. Regarding (v), the controller (16) satisfies (14a) if $\bar{\omega}_{i}^{\text {th }}<\omega_{i} \leqslant \bar{\omega}_{i}$, and satisfies (14b) if $\underline{\omega}_{i} \leqslant \omega_{i}<\underline{\omega}_{i}^{\text {th }}$; hence by Lemma 4.4 both $\overline{\mathscr{C}}_{i}$ and $\underline{\mathscr{C}}_{i}$ are invariant.

Proving monotonicity in $(v i)$ is equivalent to showing that $\dot{\omega}_{i}(t) \leqslant$ 0 when $\omega_{i}(t)>\bar{\omega}_{i}$ and $\dot{\omega}_{i}(t) \geqslant 0$ when $\omega_{i}(t)<\underline{\omega}_{i}$. For simplicity we only prove the first case. Note that $u_{i}(x, p) \leqslant \frac{-\bar{\alpha}_{i}\left(\omega_{i}-\bar{\omega}_{i}\right)}{\left(\omega_{i}-\bar{\omega}_{i}^{\mathrm{th}}\right)}+$ $q_{i}(x, p)$. Plugging this into (4b) and using $\omega_{i}>\bar{\omega}_{i}$, one has

$$
M_{i} \dot{\omega}_{i} \leqslant \frac{-\bar{\alpha}_{i}\left(\omega_{i}-\bar{\omega}_{i}\right)}{\left(\omega_{i}-\bar{\omega}_{i}^{\mathrm{th}}\right)} \leqslant 0
$$

establishing monotonicity (notice that the inequality holds even if the initial condition does not belong to $\Phi$ ). Finally, since $\omega^{\infty} \in$ $\left(\underline{\omega}_{i}^{\text {th }}, \bar{\omega}_{i}^{\text {th }}\right)$ and $\omega_{i}(t) \rightarrow \omega^{\infty}$ for every $i \in \mathscr{I}$, there exists $t_{1}$ such that $\omega_{i}\left(t_{1}\right) \in\left[\underline{\omega}_{i}^{\text {th }}, \bar{\omega}_{i}^{\text {th }}\right]$, which, by $(v)$, further implies that $\omega(t) \in$ $\left[\underline{\omega}_{i}^{\text {th }}, \bar{\omega}_{i}^{\text {th }}\right]$ for every $t \geqslant t_{1}$.

Remark 5.4 (Performance trade-offs via selection of class- $\mathscr{K}$ functions). As pointed out in Section 4.2, the choice of class- $\mathscr{K}$ functions affects the system behavior. To illustrate this, consider the linear choice $\bar{\alpha}_{i}=\underline{\alpha}_{i}: \mathbb{R} \rightarrow \mathbb{R}, s \mapsto \gamma_{i} s$, where $\gamma_{i}>0$ is a design parameter. A smaller $\gamma_{i}$ leads to more stringent requirements on the derivative of the frequency. This is because $u_{i}(x, p)$ can be non-zero only when either of the following happen,

$$
\begin{gathered}
\frac{-\bar{\alpha}_{i}\left(\omega_{i}-\bar{\omega}_{i}\right)}{\left(\omega_{i}-\bar{\omega}_{i}^{\text {th }}\right)}+q_{i}(x, p)<0 \text { and } \omega_{i}>\bar{\omega}_{i}^{\text {th }}, \\
\frac{\underline{\alpha}_{i}\left(\underline{\omega}_{i}-\omega_{i}\right)}{\underline{\omega}_{i}^{\text {th }}-\omega_{i}}+q_{i}(x, p)>0 \text { and } \omega_{i}<\underline{\omega}_{i}^{\text {th }} .
\end{gathered}
$$

In this first case, the term $\frac{-\bar{\alpha}_{i}\left(\omega_{i}-\bar{\omega}_{i}\right)}{\left(\omega_{i}-\bar{\omega}_{i}^{\text {th }}\right)}=\frac{\gamma_{i}\left(\bar{\omega}_{i}-\omega_{i}\right)}{\omega_{i}-\bar{\omega}_{i}^{\text {th }}}>0$ becomes smaller as $\gamma_{i}$ decreases, making its addition with $q_{i}(x, p)$ more likely to be less than 0 , and resulting in an earlier activation of $u_{i}$. The second case follows similarly.

A small $\gamma_{i}$ may also lead to high control magnitude because it prescribes a smaller bound on the frequency derivative, which in turn may require a larger control effort. However, choosing a large $\gamma_{i}$ may cause the controller to be highly sensitive to $\omega_{i}$. This is because the absolute value of the partial derivative of $\frac{-\bar{\alpha}_{i}\left(\underline{\omega}_{i}-\omega_{i}\right)}{\left(\omega_{i}-\bar{\omega}_{i}^{\mathrm{th}}\right)}$ (resp. $\left.\frac{\underline{\alpha}_{i}\left(\underline{\omega}_{i}-\omega_{i}\right)}{\underline{\omega}_{i}^{\text {th }}-\omega_{i}}\right)$ with respect to $\omega_{i}$ grows proportionally with $\gamma_{i}$; consequently, when $u_{i}(x, p)$ is non-zero, its sensitivity against $\omega_{i}$ increases as $\gamma_{i}$ grows, resulting in low tolerance against slight changes in $\omega_{i}$. In the limit, as $\gamma_{i} \rightarrow \infty$, this yields

$$
u_{i}^{\infty}(x, p)= \begin{cases}\min \left\{0, q_{i}(x, p)\right\} & \omega_{i}=\bar{\omega}_{i}, \\ 0 & \underline{\omega}_{i}<\omega_{i}<\bar{\omega}_{i}, \\ \max \left\{0, q_{i}(x, p)\right\} & \omega_{i}=\underline{\omega}_{i},\end{cases}
$$

which in general is discontinuous. We illustrate in simulation the dependence of the controller on the choice of linear class- $\mathscr{K}$ functions in Section 7.

\section{Closed-loop performance analysis}

In this section, we characterize additional properties of the closedloop system under the proposed distributed controller beyond stability and frequency invariance. We characterize the attractivity rate of trajectories for initial conditions outside the safe frequency region, the boundedness of the control effort prescribed by the controller along the system trajectories, and its robustness against measurement and parameter uncertainty.

\subsection{Estimation of the attractivity rate}

Here we provide an estimate of the convergence rate to the safe region (cf. Theorem 5.3(vi)) when the frequency of a node is initially outside it. The next result identifies a specific trajectory bounding the frequency evolution.

Lemma 6.1 (Upper bound on frequency evolution). With the notation of Theorem 5.3, assume that for some $i \in \mathfrak{C}, \omega_{i}(0)>\bar{\omega}_{i}$. Let $z_{i}(t)$ be the unique solution of

$$
M_{i} \dot{z}_{i}(t)=\frac{-\bar{\alpha}_{i}\left(z_{i}(t)-\bar{\omega}_{i}\right)}{z_{i}(t)-\bar{\omega}_{i}^{\text {th }}}, z_{i}(0)=\omega_{i}(0) .
$$

Then it holds that $\omega_{i}(t) \leqslant z_{i}(t)$, for any $t \geqslant 0$. Furthermore, $z_{i}(t)$ converges to $\bar{\omega}_{i}$ monotonically without reaching it in finite time.

PROOF. It is easy to check that if $z_{i}(0)>\bar{\omega}_{i}$, then there exists a unique solution of (20) for every $t \geqslant 0$. Since (18) holds for every 
$i \in \mathfrak{C}$, by the Comparison Lemma [Khalil, 2002, Lemma 3.4], one has that $\omega_{i}(t) \leqslant z_{i}(t)$ for any $t \geqslant 0$. On the other hand, one can easily prove via Lemma 2.1 that the set $\left\{z_{i} \mid \bar{\omega}_{i}-z_{i} \leqslant 0\right\}$ is invariant, which, together with the fact that $z_{i}(0)>\bar{\omega}_{i}$, implies $z_{i}(t) \geqslant \omega_{i}$ for every $t \geqslant 0$. By the dynamics (20), we deduce $\dot{z}_{i}(t) \leqslant$ 0 for every $t \geqslant 0$ and the monotonicity follows. Finally, since $z_{i}(t)$ is monotone decreasing and lower-bounded, $z_{i}(t)$ is convergent, with limit $\bar{\omega}_{i}$ (since $\dot{z}_{i}(t)<0$ if $z_{i}(t) \neq \bar{\omega}_{i}$ ). Finally, since the uniqueness of trajectories is guaranteed by the Lipschitzness of the dynamics (20) and $\bar{\omega}_{i}$ is an equilibrium, it follows that $z_{i}(t)>$ $\bar{\omega}_{i}$ for any $t \geqslant 0$.

A similar statement holds for the case when the initial frequency is lower than the lower safe bound, but we omit it for brevity. When $\bar{\alpha}_{i}$ is linear, the next result provides an explicit expression for the bounding trajectory.

Corollary 6.2 (Estimation of frequency convergence rate with linear class- $\mathscr{K}$ function). With the notation of Lemma 6.1, if $\bar{\alpha}_{i}(s)=\bar{\gamma}_{i} s$ with $\bar{\gamma}_{i}>0$, then $z_{i}(t)$ is uniquely determined by

$$
z_{i}(t)+\left(\bar{\omega}_{i}-\bar{\omega}_{i}^{\mathrm{th}}\right) \ln \left(\frac{z_{i}(t)-\bar{\omega}_{i}}{\omega_{i}(0)-\bar{\omega}_{i}}\right)=-\bar{\gamma}_{i} t / M_{i}+\omega_{i}(0) .
$$

Furthermore, it holds that for any $t \geqslant 0$,

$$
z_{i}(t) \leqslant \bar{\omega}_{i}+\left(\omega_{i}(0)-\bar{\omega}_{i}\right) \exp \left(\frac{-\bar{\gamma}_{i} t / M_{i}+\omega_{i}(0)-\bar{\omega}_{i}}{\bar{\omega}_{i}-\bar{\omega}_{i}^{\text {th }}}\right) .
$$

PROOF. In the case where $\bar{\alpha}_{i}(s)=\bar{\gamma}_{i} s$, by separation of variables, one has that (20) is equivalent to

$$
\frac{z_{i}-\bar{\omega}_{i}^{\text {th }}}{z_{i}-\bar{\omega}_{i}} \mathrm{~d} z_{i}=-\bar{\gamma}_{i} \mathrm{~d} t / M_{i}, z_{i}(0)=\omega_{i}(0) .
$$

Equation (21) follows by integrating the above differential equation. Since by Lemma $6.1 z_{i}(t) \geqslant \bar{\omega}_{i}$ for every $t \geqslant 0$, it holds

$$
\bar{\omega}_{i}+\left(\bar{\omega}_{i}-\bar{\omega}_{i}^{\mathrm{th}}\right) \ln \left(\frac{z_{i}(t)-\bar{\omega}_{i}}{\omega_{i}(0)-\bar{\omega}_{i}}\right) \leqslant-\bar{\gamma}_{i} t / M_{i}+\omega_{i}(0),
$$

concluding the proof.

Remark 6.3 (Estimation of safe-frequency entry time). Corollary 6.2 establishes the exponential convergence rate of the frequency evolution to the safe region, but it does not provide an estimate of the finite time of entry $t_{1}$ stated in Theorem 5.3(vi). This is because the upper-bound signal $z_{i}$ never hits $\bar{\omega}_{i}$ in finite time. This drawback is caused by the fact that the existence of $t_{1}$ is justified by (cf. proof of Theorem 5.3(vi)) the combination of frequency invariance and convergence of the closed-loop system, where we do not utilize the latter in obtaining the upper-bound signal. To fix this, one may replace $\bar{\omega}_{i}$ by $\bar{\omega}_{i}-\varepsilon_{i}$ in (16) with $\varepsilon_{i} \in \mathbb{R}_{>}$, and determine $t_{1}$ by solving $z\left(t_{1}\right)=\bar{\omega}_{i}$ along the dynamics (20). Note that, although this procedure does not jeopardize any statement in Theorem 5.3, it actually puts a stricter frequency invariance requirement on the controller.

\subsection{Bounds on controller magnitude}

Here, we provide bounds on the amplitude of the proposed controller (16) along the system trajectories for a given constant power injection profile $p$. Our approach to do this is to constrain the allowable initial conditions by employing the energy function $V$ as a measure of how far an initial state can be from the equilibrium point. Formally, let

$$
\hat{\Phi}(\eta) \triangleq\left\{(\lambda, \omega) \mid \lambda \in \Gamma_{\mathrm{cl}}, V(\omega, \lambda) \leqslant \eta\right\}
$$

be the collection of allowable initial states, where $0 \leqslant \eta<c$. The next result bounds the control input as a function of $\eta$.

Lemma 6.4 (Lower bound on control effort). For $i \in \mathfrak{C}$, let $g_{i}(\lambda, \omega) \triangleq \frac{-\bar{\alpha}_{i}\left(\omega_{i}-\bar{\omega}_{i}\right)}{\omega_{i}-\bar{\omega}_{i}^{\mathrm{th}}}+q_{i}(x, p)$ and $d_{i} \triangleq 1 / 2 M_{i}\left(\omega_{i}^{\mathrm{th}}-\omega^{\infty}\right)^{2}$. Let $\left(\lambda^{*}, \omega^{*}\right)$ be the optimal solution of

$\begin{array}{ll}\min _{(\lambda, \omega)} & g_{i}(\lambda, \omega) \\ \text { s.t. } & (\lambda, \omega) \in \hat{\Phi}(\eta) \\ & \lambda \in \operatorname{range}(D) \\ & \omega_{i}>\omega_{i}^{\text {th }}\end{array}$

and define

$$
u_{i}^{\min }(\eta) \triangleq \begin{cases}0 & \text { if } 0 \leqslant \eta \leqslant d_{i} \\ \min \left\{0, g_{i}\left(\lambda^{*}, \omega^{*}\right)\right\} & \text { if } d_{i}<\eta<c .\end{cases}
$$

Then, for any $(\lambda(0), \omega(0)) \in \hat{\Phi}(\eta)$ with $\lambda(0) \in \operatorname{range}(D)$,

$$
u_{i}(x(t), p) \geqslant u_{i}^{\min }(\eta)
$$

for any $t \geqslant 0$, and there exists initial states such that equality holds at some $t \geqslant 0$.

PROOF. Note that by Theorem 5.3 with $\beta=c / \eta>1$, one has $(\lambda(t), \omega(t)) \in \hat{\Phi}(\eta)$ and $\lambda(t) \in \operatorname{range}(D)$ for every $t>0$, provided they hold at $t=0$. Therefore, to show (24) for every $t \geqslant 0$, it suffices to show it holds for $t=0$. If $0 \leqslant \eta \leqslant d$, then $1 / 2 M_{i}\left(\omega_{i}(0)-\omega^{\infty}\right)^{2} \leqslant V(\omega(0), \lambda(0)) \leqslant d_{i}=1 / 2\left(M_{i}\left(\omega_{i}^{\text {th }}-\right.\right.$ $\left.\omega^{\infty}\right)^{2}$, which implies $\omega_{i}(0) \leqslant \omega_{i}^{\text {th }}$; therefore, $u_{i}(x(0), p) \geqslant 0$ follows by (16). Also, $u_{i}(x(0), p)$ can be 0 in the case when, say, $x(0)=\left(\lambda^{\infty}, \omega^{\infty}\right)$. In the other case, if $d_{i}<\eta<c$, then $u_{i}(x(0), p)$ is lower bounded by the optimal value of

$$
\begin{array}{lll}
(\hat{\mathbf{Q}}) \quad \min _{\lambda, \omega} & u_{i}(x, p) \\
& \text { s.t. } & (22 \mathrm{a}) \text { and }(22 \mathrm{~b}) .
\end{array}
$$

Denote this optimal value by $v_{i}(\eta)$. Also, the value of $u_{i}(x(0), p)$ can be exactly $v_{i}(\eta)$, e.g., in the case when $x(0)$ is the optimal solution of $(\hat{Q})$. Note that $v_{i}(\eta) \leqslant 0$ as $\left(\lambda^{\infty}, \omega^{\infty}\right)$ satisfies (25) and $u_{i}\left(\left(\lambda^{\infty}, \omega^{\infty}\right), p\right)=0$. Since it holds that a) $u_{i}(x, p) \geqslant 0$ for any $\omega_{i} \leqslant \omega_{i}^{\text {th }}$, and b) $u_{i}(x, p) \leqslant 0$ for any $\omega_{i} \geqslant \omega_{i}^{\text {th }}$, one can, without changing the optimal value, replace $u_{i}(x, p)$ by $\min \left\{0, g_{i}(\lambda, \omega)\right\}$ in $(\hat{Q})$, and meanwhile add an additional constraint (22c). With a simple reasoning effort, one can show that for this new optimization problem, the optimal value is exactly $\min \left\{0, g_{i}\left(\lambda^{*}, \omega^{*}\right)\right\}$.

Note that the control amplitude lower bound $u_{i}^{\min }(\eta)$ depends nonlinearly on the power injection $p$. This is because, although the objective function in the optimization problem $(Q)$, linearly depends on $p$, the optimal value does depend nonlinearly on $p$ through the constraint (22a). This is due to the fact that the 
equilibrium $\left(\lambda^{\infty}, \omega^{\infty} \mathbf{1}_{n}\right)$ depends on $p$ through the transcendental equation (6).

A similar result can be stated regarding an upper bound of the controller magnitude, but we omit it for brevity. The problem $(Q)$ is non-convex due to the non-convexity of the objective function. We next show that its optimal value equals that of another optimization problem with convex objective function and non-convex feasible set. Define the function $h_{i}: \mathbb{R}^{m+n} \times \mathbb{R} \rightarrow$ $\mathbb{R},(z, \omega) \rightarrow h_{i}(z, \omega)$ exactly the same as $g_{i}$ but replacing $\sin \lambda_{i}$ by $z_{i}$ in the definition of $q_{i}$. In this way, $h_{i}(\sin \lambda, \omega)=g_{i}(\lambda, \omega)$. Let $\mathscr{D}_{i}^{+} \triangleq\left\{j \mid\left[D^{T} Y_{b}\right]_{i j}>0\right\}$ and $\mathscr{D}_{i}^{-} \triangleq\left\{j \mid\left[D^{T} Y_{b}\right]_{i j}<0\right\}$. Consider the optimization

$$
\begin{array}{ll}
\min _{(z, \lambda, \omega)} & h_{i}(z, \omega) \\
\text { s.t. } \quad & \sin \lambda_{j} \leqslant z_{j}, \forall j \in \mathscr{D}_{i}^{+}, \\
& \sin \lambda_{j} \geqslant z_{j}, \forall j \in \mathscr{D}_{i}^{-}, \\
& (22 \mathrm{a}) \text { to }(22 \mathrm{c}),
\end{array}
$$

We claim that the optimal value of this problem is the same as that of $(Q)$. The claim holds if every optimal solution of $(R)$, denoted by $\left(z^{\sharp}, \lambda \sharp, \omega^{\sharp}\right)$, satisfies (26a) and (26b) with equality signs. This has to be the case since, for instance, if $\sin \lambda_{k}^{\sharp}<z_{k}^{\sharp}$ for some $k \in \mathscr{D}_{i}^{+}$, then $\left(z^{\sharp}, \lambda^{\sharp}, \omega^{\sharp}\right)$ can no more be an optimal solution, since $\left(\hat{z}^{\sharp}, \lambda^{\sharp}, \omega^{\sharp}\right)$, where $\hat{z}^{\sharp}$ differs from $z^{\sharp}$ only in its $k$ th component, $\hat{z}_{k}^{\sharp}=\sin \lambda_{k}^{\sharp}$, has $h_{i}\left(\hat{z}^{\sharp}, \omega^{\sharp}\right)<h_{i}\left(z^{\sharp}, \omega^{\sharp}\right)$, violating optimality.

Our next step is to convexify $(R)$. Here we assume that $\omega_{i} \mapsto$ $\frac{-\bar{\alpha}_{i}\left(\omega_{i}-\bar{\omega}_{i}\right)}{\omega_{i}-\bar{\omega}_{i}^{\text {th }}}$ is convex in $\omega_{i}$ in the region $\omega_{i}>\omega_{i}^{\text {th }}$, which suffices to guarantee the convexity of $(z, \omega) \mapsto h_{i}(z, \omega)$ in $(z, \omega)$ under constraint (26) (this convexity assumption holds if, for instance, $\bar{\alpha}_{i}$ is a linear function). To handle the non-convexity of the constraints (26a) and (26b), in the following two results, we separately provide inner and outer approximations, leading to upper and lower approximations of the optimal value of $(R)$, and equivalently $(Q)$.

Lemma 6.5 (Upper bound of optimal value). Define $\mathscr{H}^{+} \triangleq$ $\{(a, b)|| a \mid<\pi / 2, \sin a \leqslant b$ if $a \in[-\pi / 2,0)$, and $a \leqslant b$ if $a \in$ $[0, \pi / 2]\}$, and $\mathscr{H}^{-} \triangleq\{(a, b)|| a \mid<\pi / 2, a \geqslant b$ if $a \in[-\pi / 2,0)$, and $\sin a \geqslant b$ if $a \in[0, \pi / 2]\}$. Consider the convex optimization problem

$(\overline{\mathbf{R}})$

$$
\begin{array}{ll}
\min _{(z, \lambda, \omega)} & h_{i}(z, \omega) \\
\text { s.t. } & \left(\lambda_{j}, z_{j}\right) \in \mathscr{H}^{+}, \forall j \in \mathscr{D}_{i}^{+}, \\
& \left(\lambda_{j}, z_{j}\right) \in \mathscr{H}^{-}, \forall j \in \mathscr{D}_{i}^{-}, \\
& (22 \mathrm{a}) \text { to }(22 \mathrm{c}),
\end{array}
$$

and denote its optimal solution by $\left(z^{o}, \lambda^{o}, \omega^{o}\right)$. Then it holds that $h_{i}\left(z^{o}, \omega^{o}\right) \geqslant g_{i}\left(\lambda^{o}, \omega^{o}\right) \geqslant g_{i}\left(\lambda^{*}, \omega^{*}\right)$.

PROOF. The second inequality holds since $\left(\lambda^{o}, \omega^{o}\right)$ satisfies (22a) to (22c), making it a feasible point for $(Q)$. To show the first inequality, one can easily check that for any $j \in \mathscr{D}_{i}^{+}$, if $\left(\lambda_{j}, z_{j}\right) \in \mathscr{H}^{+}$, then $\sin \lambda_{j} \leqslant z_{j}$ (cf. Figure 1(a)). Therefore, (27a) is stricter than (26a). Similarly, (27b) is stricter than (26b). Therefore, $\left[D^{T} Y_{b}\right]_{i j} z_{j}^{o} \geqslant\left[D^{T} Y_{b}\right]_{i j} \sin \lambda_{j}^{o}$ holds for any $j \in[1, m]_{\mathbb{N}}$, completing the proof since $h_{i}\left(z^{o}, \omega^{o}\right) \geqslant$ $h_{i}\left(\sin \lambda^{o}, \omega^{o}\right)=g_{i}\left(\lambda^{o}, \omega^{o}\right)$.
Lemma 6.6 (Lower bound of optimal value). Define $\mathscr{M}_{0}^{+} \triangleq$ $\{(a, b) \mid-\pi / 2<a \leqslant 0, \sin a \leqslant b\}, \mathscr{M}_{1}^{+} \triangleq\{(a, b) \mid 0 \leqslant a \leqslant$ $\pi / 2,2 a / \pi \leqslant b\}, \mathscr{M}_{0}^{-} \triangleq\{(a, b) \mid-\pi / 2<a \leqslant 0,2 a / \pi \geqslant b\}$, and $\mathscr{M}_{1}^{-} \triangleq\{(a, b) \mid 0 \leqslant a \leqslant \pi / 2, \sin a \leqslant b\}$. Consider the convex optimization problem for $\mu \triangleq\left\{\mu_{j}\right\}_{j \in \mathscr{D}_{i}^{+} \cup \mathscr{D}_{i}^{-}}$, with $\mu_{j} \in\{0,1\}$,

$$
\begin{array}{ll}
\left(\underline{\mathbf{R}}^{\mu}\right) \quad \min _{(z, \lambda, \omega)} & h_{i}(z, \omega) \\
\text { s.t. } \quad & \left(\lambda_{j}, z_{j}\right) \in \mathscr{M}_{\mu_{j}}^{+}, \forall j \in \mathscr{D}_{i}^{+}, \\
& \left(\lambda_{j}, z_{j}\right) \in \mathscr{M}_{\mu_{j}}^{-}, \forall j \in \mathscr{D}_{i}^{-}, \\
& (22 \mathrm{a}) \text { to }(22 \mathrm{c}),
\end{array}
$$

and denote its optimal solution by $\left(\underline{z}^{\mu}, \underline{\lambda}^{\mu}, \underline{\omega}^{\mu}\right)$. Let $\mu^{*} \triangleq$ $\arg \min _{\mu} h_{i}\left(\underline{z}^{\mu}, \underline{\omega}^{\mu}\right)$, then $h_{i}\left(\underline{z}^{\mu^{*}}, \underline{\omega}^{\mu^{*}}\right) \leqslant g_{i}\left(\lambda^{*}, \omega^{*}\right)$.

PROOF. Define

$$
\begin{array}{ll}
\min _{(z, \lambda, \omega)} & h_{i}(z, \omega) \\
\text { s.t. } & \left(\lambda_{j}, z_{j}\right) \in \mathscr{M}_{0}^{+} \cup \mathscr{M}_{1}^{+}, \forall j \in \mathscr{D}_{i}^{+}, \\
& \left(\lambda_{j}, z_{j}\right) \in \mathscr{M}_{0}^{-} \cup \mathscr{M}_{1}^{-}, \forall j \in \mathscr{D}_{i}^{-}, \\
& (22 \mathrm{a}) \text { to }(22 \mathrm{c}) .
\end{array}
$$

One can easily see that (26a)-(26b) is stricter than (29a)-(29b) (cf. Figure 1(b)). Hence the optimal value of $(\underline{R})$ lower bounds $g_{i}\left(\lambda^{*}, \omega^{*}\right)$. Notice that (28a)-(28b) simply splits (29a)-(29b) into convex regions, and hence $\left(\underline{z}^{\mu^{*}}, \underline{\lambda}^{\mu^{*}}, \underline{\omega}^{\mu^{*}}\right)$ is also the optimal solution of $\left(\underline{R}^{\mu}\right)$.

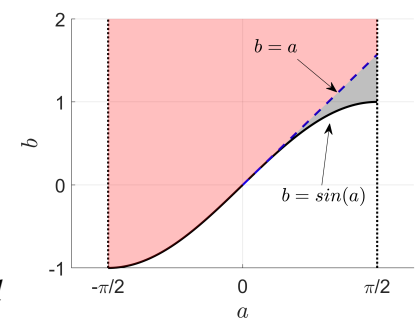

(a)

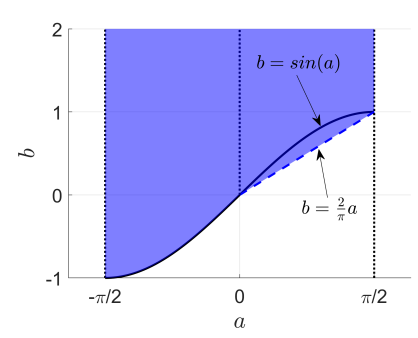

(b)
Figure 1. Tightening and relaxation of a sinusoidal non-convex constraint. In plot (a), within $|a|<\pi / 2$, by ignoring the gray region delimited by $b=a, b=\sin (a)$ and $a=\pi / 2$, the non-convex set characterized by $\sin (a) \leqslant b$ appearing in (26a) contains the red convex subset $\mathscr{H}^{+}$. On the other hand, in plot (b), this non-convex set is contained in the blue region. Each of the blue regions separated by the dotted line at $a=0$ are convex.

Together, Lemmas 6.5 and 6.6 provide us with efficient ways of approximating the value of the bound on the control effort $u_{i}^{\min }(\eta)$.

\subsection{Robustness to measurement and parameter uncertainty}

Here we study the controller performance under measurement and parameter uncertainty. This is motivated by scenarios where the state or the power injection may not be precisely measured, or scenarios where some system parameters, like the damping coefficient, are only approximately known. Formally, we let $\hat{x}=(\hat{\lambda}, \hat{\omega})$, $\hat{p}$, and $\hat{E}$ be the measured or estimated state, power injection, and 
damping parameters, respectively. For every $i \in \mathfrak{C}$, we introduce the error variables

$$
\begin{array}{ll}
\varepsilon_{i}^{\omega} \triangleq \hat{\omega}_{i}-\omega_{i}, & \varepsilon_{i}^{\lambda} \triangleq\left[D^{T} Y_{b}\right]_{i} \hat{\lambda}-\left[D^{T} Y_{b}\right]_{i} \lambda, \\
\varepsilon_{i}^{p} \triangleq \hat{p}_{i}-p_{i}, & \varepsilon_{i}^{E} \triangleq \hat{E}_{i}-E_{i} .
\end{array}
$$

We make the following assumption regarding the error.

Assumption 6.7 (Bounded uncertainties). For each $i \in \mathfrak{C}$,

(i) the uncertainties are piece-wise continuous and can be bounded by $\left|\varepsilon_{i}^{\omega}(t)\right| \leqslant \bar{\varepsilon}_{i}^{\omega},\left|\varepsilon_{i}^{\lambda}(t)\right| \leqslant \bar{\varepsilon}_{i}^{\lambda},\left|\varepsilon_{i}^{p}(t)\right| \leqslant \bar{\varepsilon}_{i}^{p}$, and $\left|\varepsilon_{i}^{E}(t)\right| \leqslant \bar{\varepsilon}_{i}^{E}$ for all $t \geqslant 0$;

(ii) $\omega^{\infty} \in\left(\underline{\omega}_{i}^{\text {th }}+\bar{\varepsilon}_{i}^{\omega}, \bar{\omega}_{i}^{\text {th }}-\bar{\varepsilon}_{i}^{\omega}\right)$;

(iii) $\bar{\varepsilon}_{i}^{\omega}<\min \left\{\bar{\omega}_{i}-\bar{\omega}_{i}^{\text {th }}, \underline{\omega}_{i}^{\text {th }}-\underline{\omega}_{i}\right\}$.

Condition (i) provides uniform bounds on the uncertainties; (ii) ensures that, even with uncertainty, the control input is identically 0 around the equilibrium; (iii) guarantees that the control input is always non-singular.

For convenience, we use $\hat{u}_{i}(\hat{x}, \hat{p}(t))$ to refer to the controller with the same functional expression as (16) but implemented with approximate parameter values and evaluated at the inaccurate state $\hat{x}$ and power injection $\hat{p}(t)$. Notice that $\hat{p}(t)$ can be time-varying. The next result shows that $\hat{u}_{i}$ still stabilizes the power network and enforces the satisfaction of a relaxed frequency invariance condition. For simplicity, we restrict our attention to linear class$\mathscr{K}$ functions in the controller design.

Proposition 6.8 (Robust stability and frequency invariance under uncertainty). Under condition (5) and Assumption 6.7, consider the evolution of the system (4) with the controller $\hat{u}_{i}$ for each $i \in \mathfrak{C}$. Then the following results hold provided $\lambda(0) \in \operatorname{range}(D)$ and $(\lambda(0), \omega(0)) \in \Phi$ for some $\beta>1$ :

(i) The solution exists and is unique for every $t \geqslant 0$.

(ii) $\lambda(t) \in \operatorname{range}(D)$ and $(\lambda(t), \omega(t)) \in \Phi$ for any $t \geqslant 0$;

(iii) $\left(\lambda^{\infty}, \omega^{\infty} \mathbf{1}_{n}\right)$ is stable, and $(\lambda(t), \omega(t))$ converges to $\left(\lambda^{\infty}, \omega^{\infty} \mathbf{1}_{n}\right)$

(iv) There exists a finite time $t_{2}$ such that $\hat{u}_{i}(\hat{x}(t), \hat{p}(t))=0$ for every $t \geqslant t_{2}$ and every $i \in \mathfrak{C}$.

(v) Suppose $\bar{\alpha}_{i}(s)=\underline{\alpha}_{i}(s)=\gamma_{i}$ f for every $i \in \mathfrak{C}$. Then, if there exists $\Delta>0$ such that satisfy

$$
\begin{aligned}
& \frac{-\gamma_{i}\left(\bar{\varepsilon}_{i}^{\omega}+\Delta\right)}{\bar{\omega}_{i}-\bar{\omega}_{i}^{\text {th }}+\Delta+\bar{\varepsilon}_{i}^{\omega}}+\bar{\varepsilon}_{i}^{E}\left(\Delta+\bar{\omega}_{i}\right)+\hat{E}_{i} \bar{\varepsilon}_{i}^{\omega}+\bar{\varepsilon}_{i}^{\lambda}+\bar{\varepsilon}_{i}^{p} \leqslant 0, \\
& \frac{-\gamma_{i}\left(\bar{\varepsilon}_{i}^{\omega}+\Delta\right)}{\underline{\omega}_{i}^{\text {th }}-\underline{\omega}_{i}+\Delta+\bar{\varepsilon}_{i}^{\omega}}+\bar{\varepsilon}_{i}^{E}\left(\Delta-\underline{\omega}_{i}\right)+\hat{E}_{i} \bar{\varepsilon}_{i}^{\omega}+\bar{\varepsilon}_{i}^{\lambda}+\bar{\varepsilon}_{i}^{p} \leqslant 0,
\end{aligned}
$$

then $\omega_{i}(t) \in\left[\underline{\omega}_{i}-\Delta, \bar{\omega}_{i}+\Delta\right]$ for all $t>0$, provided $\omega_{i}(0) \in$ $\left[\underline{\omega}_{i}-\Delta, \bar{\omega}_{i}+\Delta\right]$, and, if $\omega_{i}(0) \notin\left[\underline{\omega}_{i}-\Delta, \bar{\omega}_{i}+\Delta\right]$, then there exists a finite time $t_{3}$ such that $\omega_{i}(t) \in\left[\underline{\omega}_{i}-\Delta, \bar{\omega}_{i}+\Delta\right]$ for all $t \geqslant t_{3}$.

PROOF. The proofs of (i)-(iii) follow similar arguments as the proofs of Theorem (i)-(iii). For stability, one can show that $\frac{d}{d t} V(\omega(t), \lambda(t))=-\tilde{\omega}^{T}(t) E \tilde{\omega}(t)+\sum_{i \in \mathfrak{C}} \widetilde{\omega}_{i}(t) \hat{u}_{i}(\hat{x}(t), \hat{p}(t))$. By Assumption 6.7 and the definition of $\hat{u}_{i}$, it holds that $\sum_{i \in \mathfrak{C}} \tilde{\omega}_{i}(t) \hat{u}_{i}(\hat{x}(t), \hat{p}(t)) \leqslant 0$, implying $\frac{d}{d t} V(\lambda(t), \omega(t)) \leqslant 0$. The convergence follows by LaSalle Invariance Principle and noticing that $\hat{u}_{i}(\hat{x}, \hat{p}(t))$ is identically 0 so long as $\omega_{i} \in\left[\underline{\omega}_{i}^{\text {th }}+\bar{\varepsilon}_{i}^{\omega}, \bar{\omega}_{i}^{\text {th }}-\bar{\varepsilon}_{i}^{\omega}\right]$, which, together with the convergence, implies that $\hat{u}_{i}(\hat{x}(t), \hat{p}(t))$ is 0 after a finite time. For $(v)$, to prove the invariance of

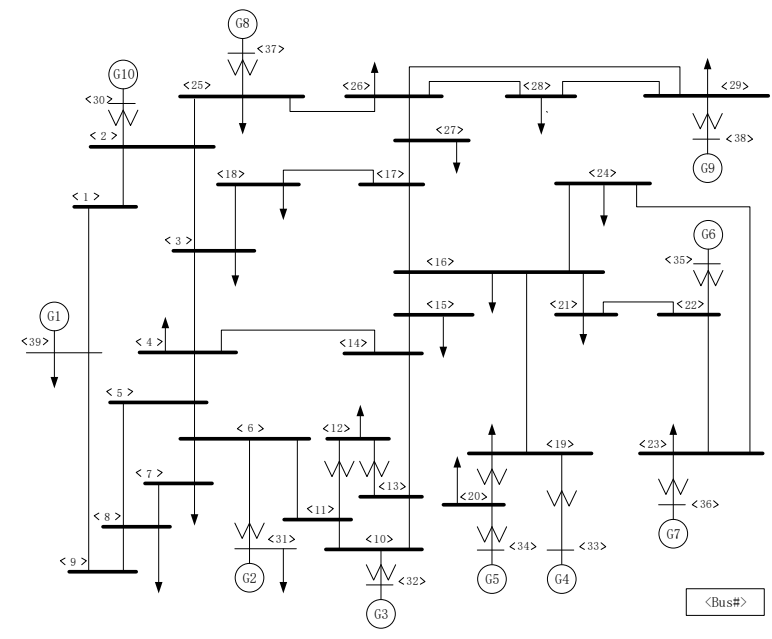

Figure 2. IEEE 39-bus power network.

$\left[\underline{\omega}_{i}-\Delta, \bar{\omega}_{i}+\Delta\right]$, by Lemma 4.3 , we only need to show that

$$
\begin{array}{r}
\hat{u}_{i}(\hat{x}, \hat{p}(t))-q_{i}(x, t) \leqslant 0, \text { if } \omega_{i}=\bar{\omega}_{i}+\Delta, \\
-\hat{u}_{i}(\hat{x}, \hat{p}(t))+q_{i}(x, t) \leqslant 0, \text { if } \omega_{i}=\underline{\omega}_{i}-\Delta .
\end{array}
$$

For simplicity, we only show that (30a) implies (31a) (the fact that (30b) implies (31b) follows similarly). Notice that if $\omega_{i}=$ $\bar{\omega}_{i}+\Delta$, then $\hat{u}_{i}(\hat{x}, \hat{p}(t))-q_{i}(x, t)$ equals

$$
\frac{-\gamma_{i}\left(\Delta+\varepsilon_{i}^{\omega}\right)}{\bar{\omega}_{i}-\bar{\omega}_{i}^{\text {th }}+\Delta+\varepsilon_{i}^{\omega}}+\varepsilon_{i}^{E}\left(\bar{\omega}_{i}+\Delta\right)+\hat{E}_{i} \varepsilon_{i}^{\omega}+\varepsilon_{i}^{\lambda}+\varepsilon_{i}^{p},
$$

which, by Assumption 6.7, is smaller than or equal to the lefthand side of (30a) by letting the uncertainties take their individual bounds; hence (31a) holds. Finally, the existence of $t_{3}$ follows a similar proof in Theorem (vi).

One should look at (30) as a condition that, independently of the specific realization of the uncertainty, guarantees that the invariance of the frequency interval is ensured.

\section{Simulations}

We illustrate the performance of our control design in the IEEE 39-bus power network displayed in Figure 2. The network consists of 46 transmission lines and 10 generators, serving a load of approximately $6 \mathrm{GW}$. We take the values of susceptance $b_{i j}$ and rotational inertia $M_{i}$ for generator nodes from the Power System Toolbox [Cheung et al., 2009]. We use this toolbox to assign the initial power injection $p_{i}(0)$ for every bus (although the analytical results hold for constant power injections, in simulation we have also tested the more general time-varying case). We assign all non-generator buses a uniform small inertia $M_{i}=0.1$. The damping parameter is $E_{i}=1$ for all buses. The initial state $(\lambda(0), \omega(0))$ is chosen to be the unique equilibrium with respect to the initial power injection. We implement the distributed controller in (16) in the generators with indices $\mathfrak{C}=\{30,31,32\}$ to tune their transient frequency behavior. The controller parameters are as follows: for every $i \in \mathfrak{C}$, we let $\bar{\alpha}_{i}(s)=\underline{\alpha}_{i}(s)=\gamma_{i} s$, with $\gamma_{i}=2, \bar{\omega}_{i}=-\underline{\omega}_{i}=0.2 \mathrm{~Hz}$ and $\bar{\omega}_{i}^{\text {th }}=-\underline{\omega}_{i}^{\text {th }}=0.1 \mathrm{~Hz}$. The nominal frequency is $60 \mathrm{~Hz}$, and hence the safe frequency region is $[59.8 \mathrm{~Hz}, 60.2 \mathrm{~Hz}]$. 


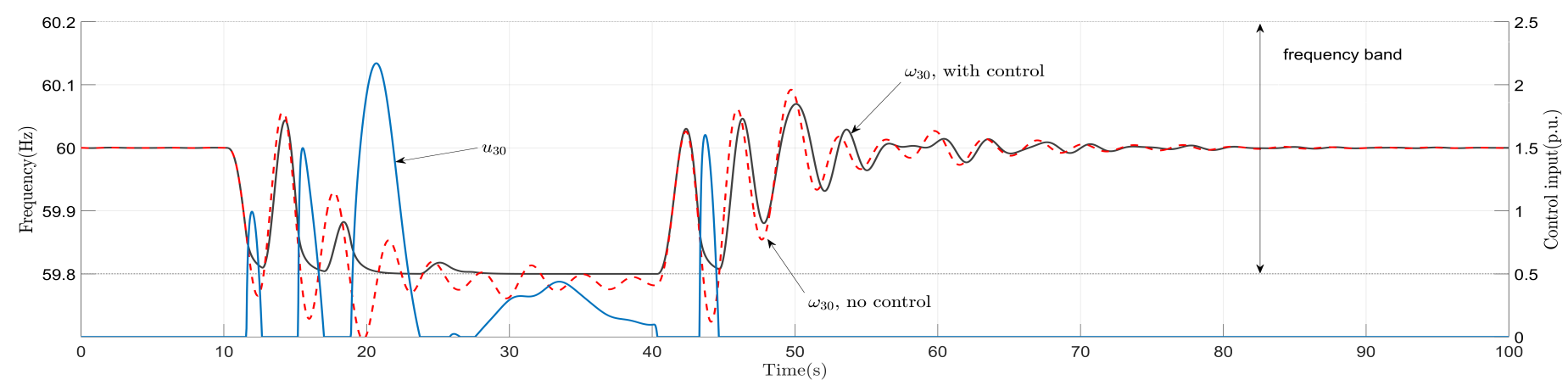

Figure 3. Frequency and control input trajectories at node 30 corresponding to the power supply loss of generator G9 during [10,40]s. The frequency trajectory without transient controller goes beyond the safe bounds during the contingency, while this is avoided with the proposed controller. Notice that the latter xonly takes effect when the frequency is close to the safe bound.

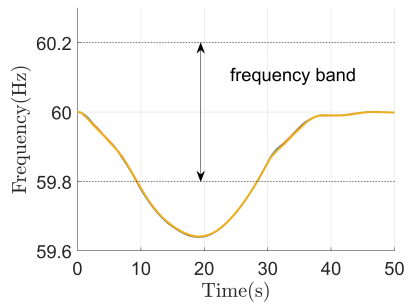

(a)

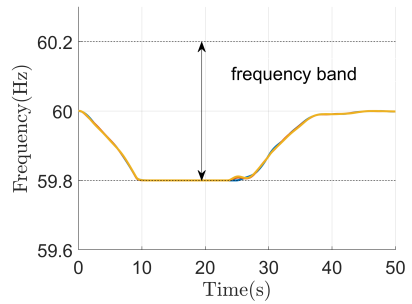

(b)

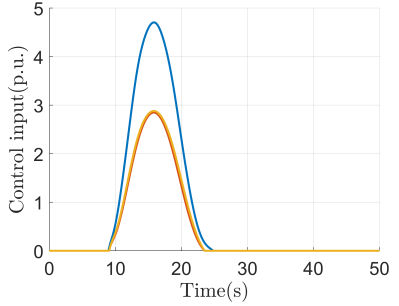

(c)



(d)

Figure 4. Frequency and control input trajectories with and without transient controller. Plot (a) shows the frequency trajectories of the generators 30, 31, and 32 without the transient controller (16), with all of them going beyond the lower safe frequency bound. With the transient controller, plot (b) shows that all frequency trajectories stay within the safe bound. Plot (c) shows the corresponding trajectories of the control inputs. Plot (d) shows the controller performance under parameter uncertainty and errors in the power injection approximation.

We first show how the proposed controller maintains the targeted generator frequencies within the safe region provided that these frequencies are initially in it. For our first scenario, we consider a generator loss and recovery process. Specifically, we set the power injection of node 38 to zero (i.e., generator G9) during the time interval $[10,40] \mathrm{s}$. As shown in Figure 3, without the transient controller (16), the frequency of node 30 first gradually goes down, exceeding the safe bound $59.8 \mathrm{~Hz}$ a few times, even tending to converge to a frequency below it. As node 38 recovers its power supply at $40 \mathrm{~s}$, the frequency comes back to $60 \mathrm{~Hz}$. In comparison, with the transient controller, the frequency trajectory never goes beyond $59.8 \mathrm{~Hz}$ during the transient.

For our second scenario, we perturb all non-generator nodes by a sinusoidal power injection whose magnitude is proportional to the corresponding node's initial power injection. Specifically, for every $i \in\{1,2, \cdots, 29\}$,

$$
p_{i}(t)= \begin{cases}p_{i}(0) & \text { if } t \geqslant 30 \\ \left(1+0.3 \sin \left(\frac{\pi t}{30}\right)\right) p_{i}(0) & \text { otherwise }\end{cases}
$$

For $i \in\{30,31, \cdots, 39\}, p_{i}(t)$ remains constant all the time. Figure 4(a) shows the frequency responses of generators 30, 31, and 32 without the transient controller. One can see that all trajectories exceed the $59.8 \mathrm{~Hz}$ lower frequency bound. For comparison, Figure 4(b) shows the trajectories with the transient controller (16), where all remain within the safe frequency region. Figure 4(c) displays the corresponding input trajectories, which converge to 0 in finite time, as stated in Theorem 5.3(iv). We also illustrate the robustness of the controller against uncertainty. We have each controller employ $\hat{E}_{i}=2$ and $\hat{p}_{i}(t)=1.1 p_{i}(t)$, corresponding to $100 \%$ and $10 \%$ deviations on droop coefficients and power in- jections, respectively. Figure 4(d) illustrates the frequency trajectories of the 3 controlled generators. Since condition (30) is satisfied with $\Delta=0.1 \mathrm{~Hz}$, Proposition 6.8 ensures that the invariant frequency interval is now $[59.7 \mathrm{~Hz}, 60.3 \mathrm{~Hz}]$.

Next, we examine the effect of the choice of class- $\mathscr{K}$ function on the behavior of the transient frequency. We focus our attention on bus 30 and simulate the network behavior for a linear function with $\gamma_{30}=0.1,2,10$, and $+\infty$ (the latter corresponding to the discontinuous controller in (19)). Figure 5 shows the corresponding frequency and control input trajectories for the first 30 seconds at node 30. From Figure 5(a), one can see that the frequency trajectory with $\gamma_{30}=0.1$ tends to stay away from the lower safe bound (overprotection), compared with the trajectories with $\gamma_{30}=2,10$, and $+\infty$, and this results in a larger control input, cf. Figure 5(b). As $\gamma_{30}$ increases, the control input is triggered later. On the other hand, choosing a large $\gamma_{30}$ lead to higher sensitivity, as observed in Figure 5(b), where the input trajectory with large $\gamma_{30}$ grows faster at the time when the control input first becomes non-zero. In fact, the controller with $\gamma_{30}=10$ exhibits a sharp change around $t=9 \mathrm{~s}$, similar to the discontinuous controller (19). The discontinuity of the latter is more evident under state measurements errors. In Figure 6, we run the same simulation but with $\hat{\omega}_{30}(t)=\omega_{30}(t)+0.001 \sin (200 \pi t)$ as the measured frequency. One can observe the high-frequency fluctuation in the control input trajectory around $9.4 \mathrm{~s}$ for $\gamma_{30}=+\infty$, whereas this does not happen for $\gamma_{30}=2$ due to its Lipschitz continuity character. These simulations validate the observations of Remark 5.4.

Next, we simulate the case where some of the generator frequencies are initially outside the safe region to show how the transient controller brings the frequencies back to it. We use the same 


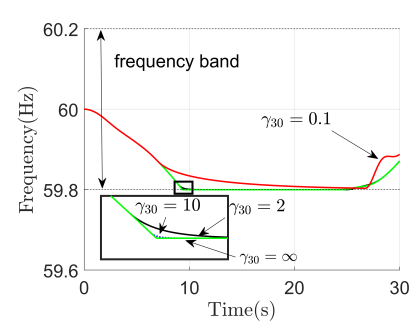

(a)



(b)
Figure 5. Frequency and control input trajectories at node 30 with linear class- $\mathscr{K}$ function with slope $\gamma_{30}=0.1,2,10$ and $+\infty$, respectively. We observe from plot (a) that the frequency trajectory with small $\gamma_{30}$ tends to stay away from the safe frequency bound, at the cost of having a large control input, as shown in plot (b). A large $\gamma_{30}$ causes the controller to be sensitive to $\omega_{30}$, making the input change rapidly around $9 \mathrm{~s}$.

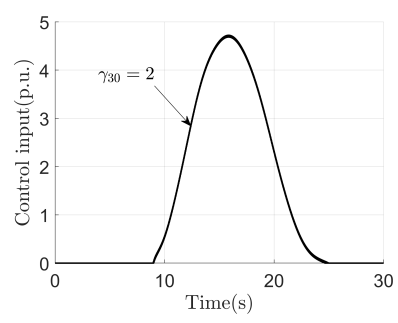

(a)

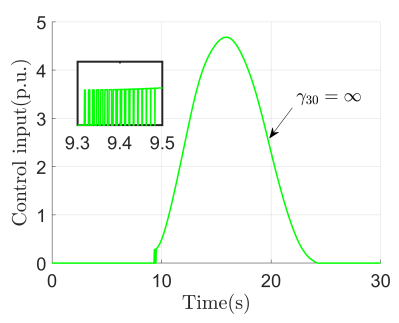

(b)
Figure 6. Control input trajectories at node 30 with linear class- $\mathscr{K}$ function with slope $\gamma_{30}=2$ and $+\infty$, respectively, under state measurement errors in $\omega_{30}$. The controller with $\gamma_{30}=2$ is Lipschitz continuous (cf. plot (a)), whereas the controller with $\gamma_{30}=+\infty$ (cf. plot (b)) is discontinuous.

setup as in Figure 4, but we only turn on the distributed controller after $t=12 \mathrm{~s}$. Figure 7(a) shows the frequency trajectories of generators 30,31 , and 32. As the controller is disabled for the first $12 \mathrm{~s}$, all 3 frequency trajectories are lower than $59.8 \mathrm{hz}$ at $t=12 \mathrm{~s}$. After $t=12 \mathrm{~s}$, all of them return to the safe region in a monotonic way, and once they are in the region, they never leave, in accordance with Theorem 5.3(vi). Figure 7(b) shows the corresponding control input trajectories.

Finally, we illustrate the bounds on control amplitude of Section 6.2. Let $\eta=0.5$ and $i=30$. By Lemma 6.4, the control input is lower bounded by $u_{i}^{\min }(\gamma)$, which requires $g_{i}\left(\lambda^{*}, \omega^{*}\right)$. The numerical computation of the upper $g_{i}\left(\lambda^{o}, \omega^{o}\right)$ (cf. Lemma 6.5) and lower $h_{i}\left(z^{\mu^{*}}, \underline{\omega}^{\mu^{*}}\right)$ (cf. Lemma 6.6) bounds both yield -5.8686 . Figure. 8(a) shows 100 input trajectories with initial states randomly selected around $\left(\lambda^{\circ}, \omega^{o}\right)$, all lower bounded by -5.8686 .

\section{Conclusions}

We have proposed a distributed transient power frequency controller that is able to maintain the nodal frequency of actuated buses within a desired safe region and to recover from undesired initial conditions. We have proven that the control input vanishes in finite time, so that the closed-loop system possesses the same equilibrium and local stability and convergence guarantees as the open-loop one. We have characterized the smoothness and robustness properties of the proposed controller. Future work will investigate the incorporation of economic cost, taking advantage of the trade-offs in the choice of class- $\mathscr{K}$ functions for controller design, the optimization of control effort by having controlled nodes have access to information beyond their immediate neigh-

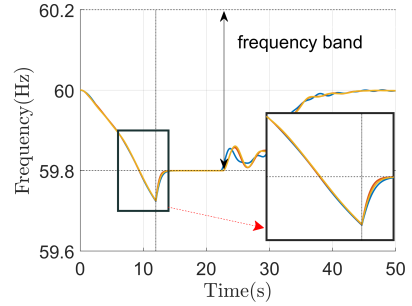

(a)

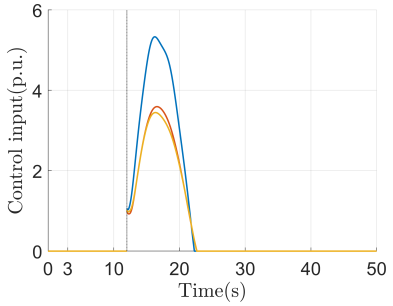

(b)
Figure 7. Frequency and control input trajectories with transient controller available after $t=12 \mathrm{~s}$. Plot (a) shows the frequency trajectories of generators 30, 31, and 32. Due to the disturbance, and without the transient controller, all 3 frequency trajectories exceed the $59.8 \mathrm{~Hz}$ safe bound at $t=12 \mathrm{~s}$. As the transient controller kicks in, the unsafe trajectories come back to the safe region and never leave afterwards. Plot (b) shows the control input trajectories.

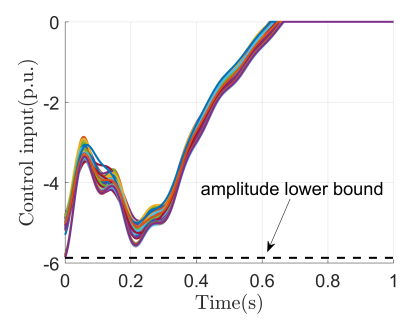

(a)

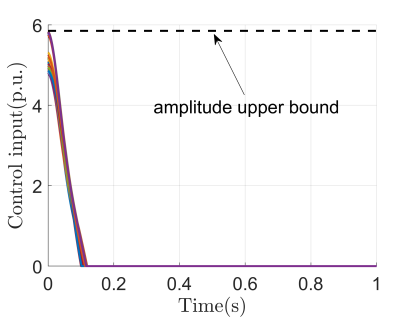

(b)
Figure 8. Control input trajectories at node 30 corresponding to 100 different initial states. In plot (a), with all initial states randomly selected around the worst-case scenario, all 100 trajectories are lower bounded by -5.8686 (denoted by the dashed line), as guaranteed by Lemma 6.4 . A similar result is illustrated in plot (b), where another 100 trajectories with random initial states are upper bounded by 5.8494 .

bors, and the understanding of the connection between actuation effort and network connectivity.

\section{References}

A. Alam and E.B. Makram. Transient stability constrained optimal power flow. In IEEE Power and Energy Society General Meeting, Montreal, Canada, June 2006. Electronic proceedings.

M. Althoff. Formal and compositional analysis of power systems using reachable sets. IEEE Transactions on Power Systems, 29(5):22702280, 2014.

A. D. Ames, X. Xu, J. W. Grizzle, and P. Tabuada. Control barrier function based quadratic programs for safety critical systems. IEEE Transactions on Automatic Control, 62(8):3861-3876, 2017.

N. Biggs. Algebraic Graph Theory. Cambridge University Press, 2 edition, 1994. ISBN 0521458978.

F. Blancini and S. Miani. Set-theoretic Methods in Control. Birkhäuser, Boston, MA, 2008.

T. S. Borsche, T. Liu, and D. J. Hill. Effects of rotational inertia on power system damping and frequency transients. In IEEE Conf. on Decision and Control, pages 5940-5946, Osaka, Japan, 2015.

F. Bullo, J. Cortés, and S. Martínez. Distributed Control of Robotic Networks. Applied Mathematics Series. Princeton University Press, 2009. ISBN 978-0-691-14195-4. Electronically available at http: //coordinationbook. info

Y. C. Chen and A. D. Domínguez-García. A method to study the effect of renewable resource variability on power system dynamics. IEEE Transactions on Power Systems, 27(4):1978-1989, 2012.

K. W. Cheung, J. Chow, and G. Rogers. Power System Toolbox, v 3.0. Rensselaer Polytechnic Institute and Cherry Tree Scientific Software, 2009.

H. D. Chiang. Direct Methods for Stability Analysis of Electric Power 
Systems: Theoretical Foundation, BCU Methodologies, and Applications. John Wiley and Sons, 2011.

H. Choi, P. J. Seiler, and S. V. Dhople. Propagating uncertainty in power-system DAE models with semidefinite programming. IEEE Transactions on Power Systems, 32(4):3146-3156, 2016.

F. Dörfler, M. Chertkov, and F. Bullo. Synchronization in complex oscillator networks and smart grids. Proceedings of the National Academy of Sciences, 110(6):2005-2010, 2013.

R. Grunbaum and J. Pernot. Thyristor-controlled series compensation: A state of the art approach for optimization of transmission over power links. Technical report, ABB, 2001.

H. K. Khalil. Nonlinear Systems. Prentice Hall, 3 edition, 2002. ISBN 0130673897.

P. Kundur. Power System Stability and Control. McGraw-Hill, 1994. ISBN 007035958X.

P. Kundur, J. Paserba, V. Ajjarapu, G. Andersson, A. Bose, C. Canizares, N. Hatziargyriou, D. Hill, A. Stankovic, C. Taylor, T. V. Cutsem, and V. Vittal. Definition and classification of power system stability. IEEE Transactions on Power Systems, 19(2):1387-1401, 2004

M. A. Mahmud, H. R. Pota, M. Aldeen, and M. J. Hossain. Partial feedback linearizing excitation controller for multimachine power systems to improve transient stability. IEEE Transactions on Power Systems, 29:561-571, 2014.

P. J. Menck, J. Heitzig, J. Kurths, and H. J. Schellnhuber. How dead ends undermine power grid stability. Nature Communications, 5(3969): $1-8,2014$.

N. W. Miller, K. Clark, and M. Shao. Frequency responsive wind plant controls: Impacts on grid performance. In Power and Energy Society General Meeting (PESGM), pages 1-8, 2011.

T. T. Nguyen, V. L. Nguyen, and A. Karimishad. Transient stabilityconstrained optimal power flow for online dispatch and nodal price evaluation in power systems with flexible ac transmission system devices. IET Generation, Transmission \& Distribution, 5:332-346, 2011.

B. K. Poolla, S. Bolognani, and F. Dorfler. Optimal placement of virtual inertia in power grids. IEEE Transactions on Automatic Control, 2017. doi: 10.1109/TAC.2017.2703302. To appear.

P. Pouyan, P. S. Kundur, and C. W. Taylor. The anatomy of a power grid blackout-root causes and dynamics of recent major blackouts. IEEE Power and Energy Magazine, 4(5):22-29, 2006.

T. L. Vu, H. D. Nguyen, A. Megretski, J. Slotine, and K. Turitsyn. Inverse stability problem and applications to renewables integration. 2017. https://arxiv.org/pdf/1703.04491.pdf.

T. L. Vu, H. D. Nguyen, A. Megretski, J. Slotine, and K. Turitsyn Inverse stability problem and applications to renewables integration. IEEE Control Systems Letters, 2(1):133-138, 2018.

Y. Zhang and J. Cortés. Transient-state feasibility set approximation of power networks against disturbances of unknown amplitude. In American Control Conference, pages 2767-2772, Seattle, WA, May 2017.

Y. Zhang and J. Cortés. Distributed transient frequency control in power networks. In IEEE Conf. on Decision and Control, Miami Beach, FL, December 2018. To appear. 